\title{
Individualisierter Zuschnitt diskursiver Anforderung und Unterstützung
}

\section{Finetuning diskurserwerbsförderlichen Lehrerhandelns in der Unterrichtsinteraktion}

\begin{abstract}
Für die Bewältigung der sprachlich-diskursiven Anforderungen, die die Partizipation an fachlichen Unterrichtsgesprächen beinhaltet, stehen Schülerinnen und Schülern in unterschiedlichem Ausmaß Ressourcen zur Verfügung. Lehrpersonen kommt somit die Aufgabe zu, sprachlich-diskursive Anforderungen und Unterstützungsverfahren individuell zuzuschneiden. Ausgehend von einem Forschungsüberblick zu den Konzepten Scaffolding und Finetuning in der Sprach- und Diskurserwerbsforschung diskutiert der Beitrag empirische Befunde zu entsprechenden Unterstützungsverfahren in der Unterrichtsinteraktion. In einem gesprächsanalytisch-rekonstruktiven Zugriff werden zwei methodische Zugänge entwickelt und erprobt, mit denen sich der individualisierte Zuschnitt diskurserwerbsförderlichen Lehrerhandelns erfassen lässt, ein querschnittlich-interindividueller und ein mikrolängsschnittlich-intraindividueller. Auf Basis videographierten Deutsch- und Mathematikunterrichts in der Primar- und Sekundarstufe werden sodann Verfahren des Finetuning in Bezug auf (1) das Kalibrieren des Anforderungsniveaus beim initialen Setzen von Zugzwängen, (2) das Readressieren und Modifizieren von Zugzwängen und (3) die Adressierung von Zuhöreraktivitäten rekonstruiert.
\end{abstract}

Keywords: Adaptivität, Scaffolding, Finetuning, Rezipientenzuschnitt, Diskurskompetenz, sprachliche Heterogenität, Unterrichtsinteraktion

\section{Einleitung}

Der ursprünglich von Wood, Bruner \& Ross (1976) geprägte und in der Folge v. a. für Erwerbsmechanismen des (frühen) Spracherwerbs verwendete Begriff Scaffolding (Bruner 1983) hat in den letzten Jahren nicht nur in der Erst-, Zweit- und

Miriam Morek, Universität Duisburg-Essen, Universitätsstr. 12, 45141 Essen, miriam.morek@uni-due.de

Vivien Heller, Bergische Universität Wuppertal, Gaußstraße 20, 42119 Wuppertal, vheller@uni-wuppertal.de

Ә Open Access. (c) 2021 Miriam Morek, Vivien Heller, publiziert von De Gruyter. (c))BY-NC-ND Dieses Werk ist lizensiert unter einer Creative Commons Namensnennung - Nicht-kommerziell - Keine Bearbeitung 4.0 International Lizenz. 
Fremdsprachdidaktik (z. B. Hammond \& Gibbons 2005; Harren 2015; vgl. auch Kleinschmidt-Schinke 2018), sondern auch in den Fachdidaktiken anderer Disziplinen (z. B. Pfister, Moser \& Pauli 2015 für den Mathematikunterricht) und den Bildungswissenschaften (z. B. van de Pol, Volman, Oort \& Beishuizen 2015) große Verbreitung erfahren. Insgesamt wurde und wird unter Scaffolding mit Bezug auf Unterricht ein breites Spektrum von zum Teil wenig spezifischen Unterstützungsverfahren zur schülerseitigen Bewältigung von (sprachlich-diskursiven) Aufgaben verstanden. Insofern ließe sich teilweise gar der Eindruck gewinnen, fast ,alles im Kontext (sprach)didaktischer Hilfestellungen werde nun unter dem Schlagwort Scaffolding verhandelt.

Jenseits seiner konzeptionellen und empirischen Unterbestimmtheit ist mit dem Gebrauch des Scaffolding-Begriffs jedoch die Hoffnung und der Anspruch verbunden, fachliche und sprachliche Anforderungen für Schülerinnen und Schüler mit sehr unterschiedlichen Ausgangsbedingungen bearbeitbar zu machen. Gerade empirische Befunde, die offenlegen, dass Schülerinnen und Schüler in sehr unterschiedlichem Maße Zugriff auf außerschulische Erwerbsressourcen (vgl. zu diesem und anderen grundlegenden Konzepten das Glossar in diesem Band) für bildungssprachliche Praktiken (z. B. Erklären, Argumentieren) haben (vgl. Morek in diesem Band; Quasthoff \& Kluger in diesem Band) und über sehr heterogene diskursive Kompetenzen verfügen, weisen auf die zentrale Rolle von Unterricht als Diskurserwerbskontext hin (Heller \& Morek 2015). Schülerinnen und Schülern einen Zugang zum Erwerb diskursiver Kompetenzen zu ermöglichen, stellt einen entscheidenden Schlüssel für schülerseitige Teilhabe an Bildungsprozessen dar (vgl. auch Quasthoff, Heller \& Morek in diesem Band).

Unter dem Gesichtspunkt der individualisierten unterrichtlichen Fördermöglichkeiten sind für den vorliegenden Beitrag entsprechend Arbeiten von besonderem Interesse, die sich mit interaktionalen Unterstützungsverfahren von Lehrkräften in Unterrichtsgesprächen beschäftigen (z. B. Harren 2015; Heller \& Morek 2015; Kleinschmidt-Schinke 2018; Koole \& Elbers 2014; Smit, van Eerde \& Bakker 2013) und dabei in den Blick nehmen, wie Lernende mit sprachlichdiskursiven Anforderungen fachlicher Unterrichtsgespräche konfrontiert und bei deren Bewerkstelligung unterstützt werden. Die Frage danach, wie dies gerade im Unterricht in heterogenen und/oder inklusiven Klassen gelingen kann, so dass alle Lernenden unabhängig von ihren Ausgangsbedingungen partizipieren und ihre sprachlich-diskursiven Fähigkeiten ausbauen können, wurde bislang weder theoretisch noch empirisch hinreichend bearbeitet. Zwar sind in jüngster Zeit Arbeiten zur Adaptivität lehrerseitigen Sprach- und Gesprächshandelns im Unterricht entstanden (z. B. Pehmer, Gröschner \& Seidel 2015; Pfister, Moser \& Pauli 2015; van Driel, Slot \& Bakker 2018; KleinschmidtSchinke 2018). Diese berücksichtigen allerdings das interaktive, sequenzielle 
Zusammenspiel aus Lerneräußerungen und lehrerseitigen Anforderungs- und Unterstützungsaktivitäten nur in Ansätzen. Vereinzelt liegen aber gesprächsanalytisch orientierte Arbeiten vor (Heller \& Morek 2015; Heller 2012; Morek 2012; Koole \& Elbers 2014), die mit Blick auf einzelne Schülerinnen und Schüler lehrerseitige Verfahren interaktiven Unterstützens aufdecken. Bemerkenswerterweise zeigt sich dabei, dass oftmals genau diejenigen Lernenden in den ,Genuss` lehrerseitiger Unterstützung kommen, die ohnehin schon über gut ausgebaute Diskurskompetenzen verfügen (Heller 2017; Prediger \& Quasthoff 2017) (Matthäus-Effekt).

Eine wichtige Stellschraube, um solchen Effekten entgegenzuwirken, könnte das im Rahmen der Spracherwerbsforschung beschriebene Finetuning sein (Bruner 1983: 38). Es bezieht sich auf den lernersensitiven, die individuellen Erwerbsstände von Lernenden berücksichtigenden Einsatz entsprechender Unterstützungsaktivitäten. $\mathrm{Ob}$ sich eine individualisierte, feinabgestimmte Diskurserwerbsunterstützung tatsächlich für Unterrichtsinteraktionen sinnvoll konzipieren und empirisch zeigen lässt, ist bislang eine offene Frage. Der vorliegende Beitrag bemüht sich um erste Antworten in diesem Feld.

Ausgehend von einem Überblick zu den Konzepten Scaffolding und Finetuning in der Sprach- und Diskurserwerbsforschung (Abschnitt 2) wird skizziert, welche Befunde bislang zum Finetuning von sprachlichen Unterstützungsverfahren im Unterricht vorliegen (Abschnitt 3). Abschnitt 4 stellt sodann anhand systematisch ausgewählter Gesprächsausschnitte vor, wie ein gesprächsanalytisch und erwerbstheoretisch fundierter Zugriff auf das Finetuning im Unterricht aussehen kann. Dazu stellen wir komplementär einen querschnittlich-interindividuellen und einen mikrolängsschnittlich-intraindividuellen Zugang vor. Ziel unseres Beitrags ist es, das Konzept des Finetuning diskurserwerbsförderlichen Lehrerhandelns theoretisch in Bezug auf das unterrichtliche Setting auszuschärfen und auf Basis authentischer Gesprächsdaten aus dem Deutsch- und Mathematikunterricht (Grundschule und Sekundarstufe) exemplarisch zu beschreiben.

\section{Finetuning und Scaffolding im Sprach- und Diskurserwerb}

\subsection{Finetuning und Scaffolding in der Spracherwerbsforschung}

Die interaktionistische Spracherwerbsforschung untersucht seit den 1970er Jahren die Rolle des interaktiven Austauschs mit Bezugspersonen für die kindliche Sprachentwicklung. Die ersten Studien konzentrierten sich dabei auf die Beschaffenheit des Input. Snow (1972) beispielsweise verglich die sprachlichen Äußerungen, die 
Mütter an zwei- und zehnjährige Kinder richten. Dabei erwies sich die an Zweijährige gerichtete Sprache u. a. als syntaktisch weniger komplex und war durch Wortwiederholungen und einen weitgehenden Verzicht auf Pronominalisierungen geprägt. Der Befund, dass Bezugspersonen ihre Sprache in Abhängigkeit vom Alter des Kindes modifizieren, stützte das Argument, dass der sprachliche Input keineswegs so unstrukturiert und unbedeutend ist wie seinerzeit von nativistischen Positionen zum Spracherwerb angenommen. In der Folge wurden weitere Parameter des Input untersucht, bspw. phonetisch-phonologische Merkmale wie ein höheres Tonhöhenregister oder besonders saliente prosodische Konturen zur Sicherung der Aufmerksamkeit des Kindes (Garnica 1977). Merkmale der sog. „motherese“ (Snow 1977) bezogen sich zunächst auf den an junge Kinder gerichteten Input. Unter dem Terminus „child directed speech“ (Gallaway \& Richards 1994) wurden zunehmend auch Merkmale der an Vorschulkinder gerichteten Sprache in den Blick genommen. Sokolov (1993) zeigt beispielsweise auf Basis längsschnittlicher Daten, dass Erwachsene in ihren Folgeäußerungen gerade solche Elemente ergänzen, die das Kind in der vorangegangenen Äußerung nicht produziert hatte. Damit wurden Parameter der Sprache der Bezugsperson und des Kindes erstmals nicht lediglich korreliert, sondern es wurden Kontingenzen zwischen adjazenten Äußerungen sichtbar gemacht. Zudem wurde zunehmend deutlich, dass child directed speech weniger als fest umrissenes Register (Ferguson 1977) zu charakterisieren ist, sondern vielmehr als flexible Adaption der Wahl sprachlicher Mittel an die jeweils gezeigten Fähigkeiten des Kindes (Cross \& Morris 1980; Snow 1977). Damit vollzog sich der Wandel von einer strukturellen Betrachtung des Input im Vergleich zu kindlichen Äußerungsformen hin zu einer Modellierung des interaktiven Zusammenspiels zwischen Erwachsenen und Kindern auf unterschiedlichen Niveaus der Sprachentwicklung.

Dazu, was solche Anpassungen an das Kompetenzniveau des Kindes letztlich bedingt, existieren unterschiedliche Hypothesen. Bisherige Evidenzen sprechen vor allem für die Relevanz kognitiver Partnermodelle und eines jeweils in situ realisierten Rezipientenzuschnitts (vgl. Fischer 2016): Wie sprachliche Äußerungen in der Interaktion mit Kindern bzw. sprachlich eingeschränkten Gesprächspartner/innen zugeschnitten werden, scheint einerseits bestimmt zu werden von generalisierten Modell-Annahmen über kognitive und sprachliche Ressourcen des Gegenübers. Andererseits liefern dessen Äußerungen im Sinne konkret gezeigter Sprachfähigkeiten (Cross, Nienhuys \& Kirkman 1985) im Zug-umZug-Aufbau von Gesprächen fortwährend Hinweise etwa zu dessen vorhandenem Vorwissen und seinen sprachlichen Kompetenzen. So wird das möglich, was die Konversationsanalyse schon früh als Recipient Design bezeichnet hat (vgl. Hitzler 2013). Recipient Design bzw. Zuhörerzuschnitt bezeichnet sie als Mechanismus, durch den ,talk by a party in a conversation is constructed or designed in ways which display an orientation and sensitivity to the particular other(s) who are the 
co-participants" (Sacks, Schegloff \& Jefferson 1974: 72). Insbesondere im letztgenannten Aspekt zeigt sich die Bedeutung der produktiven Partizipation des Kindes in Erwachsenen-Kind-Interaktionen: Erst die eigene - verbale oder nonverbale - Beteiligung des Kindes ermöglicht es dem Erwachsenen, seine Beiträge und Anforderungen auf die des Kindes zuzuschneiden.

Derartige Mechanismen der Adaption werden in der Spracherwerbsforschung als Finetuning bezeichnet. Damit ist jedoch noch nicht erklärt, wie es zu Kompetenzzuwächsen kommen kann, denn der ursprünglichen These zufolge wird dem Kind ja immer nur derjenige Input angeboten, den es bereits verarbeiten kann. Snow geht deshalb weiterführend davon aus, dass das sprachliche Angebot jeweils eine etwas höhere Komplexität aufweisen muss als das bis dahin entwickelte Sprachsystem des Kindes. Finetuning beinhalte demgemäß eine "continually adjusted optimal discrepancy between the language system of the child and the language system the child is exposed to" (Snow, Perlmann \& Nathan 1987: 66; Herv. v. M.M. \& V.H.) - mit Vygotskys Worten: Anforderungen in der Zone der nächstfolgenden Entwicklung (Vygotsky 1978).

Inspiriert von der allmählichen Verschiebung einer rein sprachstrukturell orientierten Erklärung des Spracherwerbs zu stärker interaktionsorientierten Konzeptionen geht auch Bruner (1983) von der Prämisse aus, das sich der Spracherwerb nicht auf den Erwerb des Sprachsystems beschränkt. Die Vorstellung eines allein sprachsystembezogenen ,Daten-Inputs' vernachlässige, dass Interaktanten mittels Sprache miteinander handeln - dass also auch Eltern ihren Kindern nicht einfach den passenden Input für ein Sprachverarbeitungs- und Erwerbssystem liefern (s. auch Clark 2018). Vielmehr sei der Spracherwerb Teil eines übergreifenden Prozesses, in dem das Kind zu einem „member“ seiner sozialen Gruppe wird (Schieffelin 1990; Ochs 2002; Quasthoff 2011, 2015). Spracherwerb umfasst demnach den Erwerb gesellschaftlich relevanter Formate und der für ihren Vollzug erforderlichen pragmatischen Mittel (bspw. Referenzherstellung, Bitten usw.). Bei Formaten handelt es sich um sprachlich konstituierte Interaktionsroutinen, die mehr oder weniger verfestigte Abläufe und Rollen und ein erkennbares Handlungsziel beinhalten (beim ,Guckguck-da'-Format das Wiederauftauchen, vgl. Bruner \& Sherwood 1976; beim Betrachten von Bilderbüchern das Benennen eines Gegenstands, vgl. Ninio \& Bruner 1978). Bruners Untersuchungsinteresse richtet sich vor allem auf die interaktiven Verfahren, mittels derer Erwachsene Formate initiieren bzw. auf Initiativen des Kindes eingehen und es dabei unterstützen, die für die jeweilige Aktivität konstituierenden Aufgaben zu erfüllen. Das Konzept des Finetuning bezieht Bruner dementsprechend nicht auf den Input, sondern auf die steigenden Anforderungen, die Erwachsene an die produktive Beteiligung des Kindes stellen. So zeigt er beispielsweise, dass sich Erwachsene im Rahmen des Bilderbuchlesens zunächst mit einem Zeigen des Kindes auf einen gefragten Gegenstand zufrieden- 
geben; sobald das Kind jedoch eine Wortform näherungsweise produzieren kann, passen sie ihre Fragen an und verlangen konsequent eine Benennung (Ninio \& Bruner 1978; Heller \& Rohlfing 2017). Diese Art der feinabgestimmten interaktiven Unterstützung ist bei Bruner ein zentrales Element des „Language Acquisition Support System“ (kurz: LASS, Bruner 1983). Der eher monodirektional konzipierte Input weicht aus dieser Sicht einem genuin interaktiven Unterfangen: ,[. . . ] input [. . .] is not a shower of spoken language but a highly interactive affair that is shaped [...] by some sort of an adult Language Acquisition Support System.“ (Bruner 1983: 39).

Das LASS beschränkt sich entsprechend nicht lediglich auf die Unterstützung des Erwerbs lexikalischer und morpho-syntaktischer Mittel. Für das Kind besteht eine erhebliche interaktive Anforderung auch darin, die sequenzielle Abfolge der an interaktive Rollen (z. B. Rolle des Suchenden und des sich Versteckenden) gebundenen Handlungszüge eines Formats mitzuvollziehen und zunehmend zu antizipieren. Folgerichtig bezieht sich die Unterstützung des Erwachsenen auch darauf, für die Aufrechterhaltung des Ablaufformats zu sorgen - ebenfalls feinabgestimmt darauf, inwieweit das Kind die sequenzielle Ordnung bereits überblickt. Diesen Aspekt der sequenziellen Unterstützung des gemeinsamen Vollzugs einer größeren Aktivität bezeichnet Bruner als Scaffolding. Genutzt wurde dieser Begriff von Wood, Bruner \& Ross (1976) erstmals in Bezug auf Konstruktions-Aufgaben, deren Lösung eine sequenziell geordnete Folge von Handlungsschritten beinhaltet. Zeigen ließ sich, dass für deren zielführenden Vollzug der Erwachsene ein stützendes Handlungsgerüst bereitstellt, indem er das Kind, wenn nötig, mittels nonverbaler und verbaler Hinweise steuert. Analog dazu zielt das feinabgestimmte Scaffolding ${ }^{1}$ beim Vollzug von Formaten darauf, das Kind zu befähigen, mittels Sprache zu handeln, d. h. sprachliche Ressourcen funktional einzusetzen, wie Bruner in Bezug auf den Erwerb der kindlichen Referenzherstellung deutlich macht:

If one needed a demonstration of ,fine tuning“ in language acquisition, the growth of the reference format certainly provides it. The mother restricts the task to the degrees of freedom that she believes the child can handle, and once he shows signs of doing better than that, she raises the level both of her expectancies and of her demands on the child. But to concentrate entirely on the "fine-tunedness" of the mother's implicit pedagogy is really to miss the main point. For the aim of her fine tuning is certainly not refinement for its own sake. It is the achievement of functional appropriateness that she is after.

(Bruner 1983: 124; Herv. v. M.M. \& V.H.)

1 Im Rahmen seiner Arbeiten zum Spracherwerb nimmt Bruner keine klare Abgrenzung zwischen den Begriffen Scaffolding und Finetuning vor. Während sich Scaffolding stärker auf sprachlich konstituierte und mehrschrittige Spielformate $\mathrm{zu}$ beziehen scheint, bildet Finetuning ein durchgängiges Merkmal der Verfahren, die das Language Acquisition Support System ausmachen. 
Finetuning beinhaltet somit auch, dass über die Zeit betrachtet interaktive Unterstützung, die einem Kind in der Interaktion mit einer Bezugsperson zuteilwird, allmählich in dem Maße zurückgefahren wird, wie die kindlichen Fähigkeiten ansteigen, das Kind eine zunehmend aktivere Rolle in der Interaktion übernehmen und funktional angemessen agieren kann.

\subsection{Finetuning und Scaffolding in der Diskurserwerbsforschung}

Dass sich interaktive Unterstützungsverfahren in Erwachsenen-Kind-Interaktionen, wie Bruner und andere sie für den frühen Spracherwerb aufgezeigt haben, auch für den späteren Spracherwerb finden, zeigen vor allem Studien zum Erwerb diskursiver Fähigkeiten (z. B. Hausendorf \& Quasthoff 1996; Heller 2012; Heller \& Krah 2015; Kern \& Quasthoff 2007; McCabe \& Peterson 1991; Morek 2012; Zevenbergen, Holmes, Haman et al. 2016). Als Gegenstand des Erwerbs werden dabei diskursive Praktiken wie z. B. Erzählen, Erklären und Argumentieren in den Blick genommen, also sprachliche Aktivitäten, die den Umgang mit größeren, äußerungsübergreifend organisierten kommunikativen Gattungen betreffen (vgl. Morek, Heller \& Quasthoff 2017).

Solche diskursiven Gattungen weisen in mehreren Punkten Ähnlichkeit mit den von Bruner beschriebenen Formaten (Bruner 1983: 40) auf: Auch sie eröffnen komplementäre Beteiligungsrollen (z. B. Erzähler/in und Zuhörer/in), verlangen, dass bestimmte sequenzielle Schemata, d.h. Abfolgen aus sprachlich-konstituierten Teilaufgaben bearbeitet werden, und ziehen den Rückgriff auf verfestigte sprachliche Mittel bei deren Bewerkstelligung nach sich.

Auf der anderen Seite jedoch weisen diskursive Gattungen eine sehr viel größere Kontextsensitivität und Flexibilität in ihrer konkreten interaktiven und sprachlichen Ausgestaltung auf: Anders als sprachbegleitete Formate der frühen Eltern-Kind-Interaktion (vgl. Abschnitt 2.1) stehen sie nicht für sich. Vielmehr sind sie stets in umgebende, laufende Gesprächskontexte eingebettet, in deren Rahmen sie ganz unterschiedliche Inhalte bearbeiten und wechselnde kommunikative Funktionen übernehmen (z. B. ein Verstehensproblem klären; eine lustige Begebenheit beisteuern). Jedes kontextualisiert realisierte ,Exemplar‘ einer Erzählung, eines Arguments, einer Erklärung und dergleichen weist somit eine einzigartige interaktive, inhaltliche und sprachliche Gestalt auf. Aufgabe des Kindes im Erwerb ist es, sich die - von diesen konkreten Situierungen abstrahierenden - kontextfreien Ressourcen (Sacks, Schegloff \& Jefferson 1974) zum Vollzug diskursiver Gattungen anzueignen, um selbst „Äußerungspakete“ unterschiedlichen Typs (z. B. Erzählung, Erklärung, Argument) innerhalb von Gesprächen platzieren und produzieren zu können (Morek, Heller \& Quasthoff 2017). 
Erforderlich sind dafür Fähigkeiten in den drei Teildimensionen „Kontextualisierung“, „Vertextung“ und „Markierung“ (vgl. Quasthoff, Heller \& Morek in diesem Band): Es müssen erstens sequenzielle Anschlussstellen im Gespräch für solche Äußerungspakete erkannt und sequenziell angemessen fortgeführt werden (Kontextualisierung), zweitens bedarf es eines kohärenten inneren Aufbaus (Vertextung), und drittens spezifischer sprachlicher Formen, die Funktion, Typ und Aufbau des Äußerungspaketes für die Zuhörenden deutlich machen (Markierung). Insgesamt sind somit die Diskurspraktiken nicht nur Vehikel oder Mechanismus sprachlicher Erwerbsprozesse (wie etwa das Bilderbuchbetrachten für den Erwerb syntaktischer oder lexikalischer Formen), sondern stets selbst Gegenstand bzw. Ziel des Erwerbs.

Vor diesem Hintergrund müssen auch die Gesprächs- und Zuhöreraktivitäten der erwachsenen Gesprächspartner/innen beim Vollzug diskursiver Gattungen in der Interaktion mit Kindern doppelt perspektiviert werden. Hausendorf \& Quasthoff (1996) unterscheiden - anders als Bruner - in ihrer Studie zum Erzählerwerb von Kindern zwischen 5 und 14 Jahren konsequent interaktionsorientierte Wirkweisen entsprechender Aktivitäten von entwicklungsorientierten (Hausendorf \& Quasthoff 1996: 286). Nach ihrem genuin interaktionstheoretischen Verständnis sind erwachsenenseitige Verfahren des Stellens und Anpassens von Anforderungen in gemeinsamen sprachlichen Handlungssituationen stets zuallererst auf die aktuelle Bewältigung gemeinsamer Diskursanforderungen gerichtet: Bestimmte Fragen, Nachfragen und Zuhörerreaktionen sind interaktiv motiviert, also auf Austausch und Verständigung gerichtet (und nicht etwa auf Förderung der Sprachentwicklung); sie unterstützen das Kind dabei, überhaupt eine Erzählung im Gespräch anbringen und versprachlichen zu können. Erst in ontogenetischer Betrachtung wird das Nebenprodukt dieser am Kommunikationserfolg orientierten Muster, nämlich deren entwicklungsförderliche Funktion, sichtbar (vgl. ebd.: 313).

Im Einzelnen präparieren Hausendorf \& Quasthoff anhand eines umfangreichen Korpus elizitierter Erlebniserzählungen von Kindern Unterstützungsaktivitäten der erwachsenen Interaktionspartner heraus (1996: 282; vgl. mit Blick auf Unterricht: Heller \& Morek 2015: 6), die sich vor allem auf den Umgang mit globalen Zugzwängen beziehen. Bei Zugzwängen handelt es sich um Äußerungen, mit denen im Gespräch kommunikative Erwartungen mit Blick darauf etabliert werden, welcher Typ von Äußerung als nächstes folgen muss. Die Konversationsanalyse bezeichnet solche gesprächsstrukturellen Erwartungen auch als konditionelle Relevanzen oder sequenzielle Implikationen (Schegloff \& Sacks 1973). So setzt beispielsweise ein Gruß als nächsten Äußerungstyp einen Gegengruß konditionell relevant. Globale Zugzwänge zeichnen sich nun dadurch aus, dass sie ein längeres 
Äußerungspaket (bspw. explanativen oder argumentativen Typs) erwartbar machen (Quasthoff, Heller \& Morek 2017). Eben dies wird von jungen Kindern nicht ohne Weiteres erkannt und von Erwachsenen wie folgt evoziert und unterstützt:

(1) Setzen globaler Zugzwänge, die ein (narratives) Äußerungspaket des Kindes erwartbar machen (z. B. Was war denn da gerade los mit dem Lärm?),

(2) Explizieren globaler Zugzwänge (z. B. Erzähl mal, was da los war!),

(3) Lokalisieren, d.h. Vor- oder Nachschaltung lokaler Zugzwänge statt des globalen Zugzwanges (z. B. Hast du mitgekriegt, was da passiert ist?) und Als-Ob-Behandlung lokaler Antworten des Kindes als Beiträge für die globale Aktivität (z. B. ,Erzählen'),

(4) Teilweises Übernehmen bestimmter diskursiver Aufgaben bei ausbleibenden Diskursaktivitäten des Kindes (z. B. in Form erwachsenseitiger Vermutungen).

Über die genannten Verfahren können Zugzwänge auf unterschiedliche Weise gesetzt und modifiziert werden. Je nach unterstelltem Entwicklungsniveau können so die sprachlich-diskursiven Anforderungen an den kindlichen Gesprächspartner lokal vergrößert oder verkleinert werden, wie die Autoren durch den Vergleich erwachsenenseitigen Agierens gegenüber 5-, 7-, 10- und 14-jährigen Erzähler/innen zeigen: Durch die querschnittliche Betrachtung nämlich zeichnen sie nach, dass und wie der Druck zum Elaborieren einer Erzählung und die zuhörerseitige Steuerung in Abhängigkeit vom Alter des Kindes unterschiedlich stark dosiert werden (vgl. ebd.: 285). Beispielsweise findet sich nur für Sequenzen mit 5-jährigen Erzähler/innen die Voranschaltung des lokalen Zugzwangs („Hast du mitgekriegt, was da eben passiert ist?“) gefolgt von einer Explizierung des globalen Zugzwangs („Mensch, erzähl mal!“), wohingegen schon gegenüber den 7-Jährigen sowohl auf eine Wissensabfrage als auch auf die explizite Erzählaufforderung verzichtet wird (Hausendorf \& Quasthoff 1996: 212 f.). Um Erzählinteraktionen mit Kindern unterschiedlichen Alters zum kommunikativen Gelingen zu verhelfen, werden also offensichtlich intuitiv und gemäß des allgemeinen Interaktionsprinzips von Recipient Design (vgl. 2.1) unterschiedlich stark unterstützende Verfahren gewählt.

Erst in einem zweiten Schritt deuten die Autoren diesen analytischen Befund aus entwicklungsorientierter Perspektive. Sie sehen ihn als Ausdruck von Attribuierungen bestimmter Fähigkeitsniveaus, die die Erwachsenen im Rahmen einer „naive[n] oder alltägliche[n] Diskurserwerbstheorie“ (ebd.: 299f.) in Anschlag bringen:

Aus der Sicht des Erwachsenen sieht ein angemessener, d. h. kommunikativ-effektiver Beitrag zur Erfüllung der Anforderung bereits bei den 7jährigen anders aus als bei den 5jährigen und entsprechend variieren die Anforderungen, die die Erwachsenen gegenüber 5- und 
7jährigen etablieren. Die Aktivitäten des Erwachsenen sind also angepaßt an ein unterstelltes Fähigkeitsniveau des kindlichen Gegenübers. Ein solches Fähigkeitsniveau wird dem Kind in der Interaktion mit dem Erwachsenen durch dessen einschlägige Aktivitäten attribuiert. Die Attribuierung liefert also die Gewähr dafür, daß die Anforderungen des Erwachsenen nicht stereotyp über alle Altersgruppen gleich wirksam werden, sondern an den jeweiligen Fähigkeitsstand des kindlichen Interaktionspartners sensibel angepaßt werden können. Sie verhindert so gesehen sowohl eine Über- als auch Unterforderung. Sie ermöglicht es, kurz gesagt, das Kind dort abzuholen, wo es gerade steht.

(ebd. 285, Herv. v. M.M. \& V.H.)

Entscheidend ist, dass die entsprechenden Attribuierungen und Anforderungen ihren Ausgangspunkt in den interaktiv manifest werdenden „einschlägigen Aktivitäten" des kindlichen Gegenübers nehmen, also im konversationsanalytischen Sinne „locally occasioned“ (Jefferson 1978: 220) sind. Auf der Basis der laufenden Interaktion wird somit vom Erwachsenen derjenige Bereich an Anforderungen abgesteckt, der für das jeweilige kindliche Gegenüber bearbeitbar ist bzw. in der Vygotskyschen „Zone der nächstfolgenden Entwicklung“ liegt (Hausendorf \& Quasthoff 1996: 285). Darin liegt somit die Erwerbsfunktionalität der primär auf das ,Funktionieren' der gemeinsamen Interaktion gerichteten Muster. Diesem Verständnis zufolge sind Scaffolding und Finetuning zwei untrennbar miteinander verbundene Seiten derselben Medaille.

In nachfolgenden Studien konnte ein solches diskursives Scaffolding auch für andere Gattungen (Erklären: Morek 2012; Argumentieren: Heller 2012) und habitualisierte Diskurspraktiken von Familien belegt werden (Hausendorf \& Quasthoff 1996; Heller 2012; Kern \& Quasthoff 2007; Morek 2012). Entsprechende globale und lokale Zugzwänge der Eltern sind dabei darauf gerichtet, komplexe Äußerungspakete (z. B. Erklärungen, Begründungen) sequenziell einzufordern und Unterstützung bei deren Kontextualisierung und Vertextung zu liefern. Die stabil über verschiedene Interaktionssequenzen auftretenden elternseitigen Unterstützungsverfahren lassen sich bündeln zu dem Interaktionsmuster „Fordern und Unterstützen“ (Kern \& Quasthoff 2007), das konsequent abgestimmt ist auf diskursive Möglichkeiten und Grenzen des kindlichen Partners. Allerdings dokumentieren die entsprechenden diskurssozialisatorischen Arbeiten zugleich, dass ein solches diskursives Scaffolding nicht zum Repertoire aller Eltern gehört; alternative Interaktionsmuster wie „Übernehmen und Selberlösen“ und „Raumlassen und Akzeptieren“ zeichnen sich gerade durch einen fehlenden Zuschnitt auf kindseitig bereits Gekonntes und Noch-nicht-Gekonntes aus (z. B. Heller \& Krah 2015; Quasthoff \& Kluger in diesem Band). 


\section{Finetuning diskursiver Anforderung und Unterstützung im Unterrichtsgespräch}

Es ist nun insbesondere das Konzept des Scaffolding und weniger des Finetuning, das in der Folge für unterrichtliche Lernprozesse fruchtbar zu machen versucht wurde. In der bildungswissenschaftlichen, fach- und (fremd-)sprachdidaktischen Forschung finden sich sowohl Ansätze, die den ursprünglichen Scaffolding-Gedanken von Wood, Bruner \& Ross (1976) auf fachliche Lernprozesse anwenden, als auch solche, die auf sprachliche Lernprozesse fokussieren. Mit Blick auf die Schwerpunktsetzung sprachlicher Lernprozesse lassen sich wiederum grob zwei Forschungszweige ausmachen, von denen der erste Befunde zum ungesteuerten Erst- und Zweitspracherwerb aufgreift und Formen und Wirksamkeiten von Input und Feedback (z. B. „recasts“, „repetitions“, „expansions“) auf den Erwerb vor allem sprachstruktureller Fähigkeiten in stärker gesteuerten Lernsettings untersucht (Doughty 1993; Ricart Brede 2011). Im Unterschied dazu nimmt der zweite Forschungszweig sprachliche Anforderungen im Rahmen fachlichen Lernens als Ausgangspunkt. Ein im deutschsprachigen Raum stark rezipierter Ansatz, der fachliches und sprachliches Lernen $\mathrm{zu}$ verbinden sucht, stammt von Gibbons (2002) \& Hammond (Hammond \& Gibbons 2005). In Anlehnung an die systemisch-funktionale Linguistik gehen sie davon aus, dass mündliche und schriftliche Unterrichtskommunikation auf „literate talk“, einem spezifischen Register, beruhe, das sich durch eine „increasingly abstract and metaphorical language“ auszeichne. Für die Aneignung des Registers sehen sie ein Scaffolding sowohl auf der Makroebene der Unterrichtsplanung und der Mikroebene der Unterrichtsinteraktion vor. Als Mikro-Scaffolding-Strategien nennen sie (i) Anknüpfen an Vorerfahrungen, Verknüpfen mit neuen Konzepten (,linking to prior experience/pointing forward to new experiences"), (ii) Wiederholen/Kommentieren auf der Metaebene (,recapping/meta comment”), (iii) Schülerbeiträge in adäquatem Register reformulieren (,appropriating“ and "recasting of student wording into more registrally appropriate discourse”), (iv) Beiträge elizitieren und Hinweise auf erwartete Antworten geben ("cued elicitation") und (v) Elaborierungen einfordern ("increasing prospectiveness”) im Rahmen von IRE-Sequenzen (Hammond \& Gibbons 2005: 21 ff.). Damit ist eine Gruppe recht heterogener Verfahren beschrieben, die teils auf das schülerseitige Verstehen und Einordnen neuer Themen zielen und teils auf die Aneignung bildungssprachlicher lexikalischer Ressourcen oder syntaktischer Strukturen. Bislang liegt jedoch noch kein empirischer Nachweis dazu vor, ob und im Hinblick auf welche sprachlichen Fähigkeiten die Verfahren tatsächlich erwerbssupportiv sind. Des Weiteren ist noch ungeklärt, ob die Verfahren adaptiv 
eingesetzt werden müssten, um auf die heterogenen Lernvoraussetzungen von Schülerinnen und Schülern zu reagieren.

Smit \& van Eerde (2013) unternehmen den Versuch, an Vygotsky (1978) und Gibbons (2002) angelehnte Mikro-Scaffolding-Strategien empirisch zu prüfen. Die Frage, ob sich die ursprünglich auf dyadische Interaktionen bezogenen Verfahren auch auf Interaktionen in Großgruppen übertragen lassen, beantworten sie, indem sie von einer „group zone of proximal development“ (Smit, van Eerde \& Bakker 2013: 820) ausgehen, einer Zone der nächsten Entwicklung also, die eine Lerngruppe neben individuellen Unterschieden gemein hat. Auf dieser Grundlage bestimmen sie drei Kernkomponenten des „whole class scaffolding“: erstens die Diagnose des aktuellen Entwicklungsstandes („diagnosis“), zweitens die Adaptivität des Lehrerhandelns an die schülerseitigen Voraussetzungen (,responsiveness“ bzw. „contingency“) und drittens die sukzessive Reduktion von Unterstützung („handover to independence“). Jede dieser Komponenten soll sowohl bei der vorgängigen Unterrichtsplanung als auch beim Mikro-Scaffolding zum Tragen kommen können. Mit den Komponenten wird der Aspekt des Finetuning theoretisch aufgegriffen: Scaffolding soll sich kontingent an schülerseitige Kompetenzniveaus anpassen; Voraussetzung dafür sei eine entsprechende Diagnostik. Empirisch wird diese Adaptivität allerdings lediglich auf Ebene der Gruppe erfasst, indem geprüft wird, ob sich im Verlauf einer mathematischen Unterrichtseinheit (zur Interpretation von Diagrammen) ein Wechsel von stark supportiven Verfahren zu schwach supportiven Verfahren beobachten lässt; zu ersteren zählen die Autoren „reformulating or extending pupils spoken or written utterances“ und „explicitly/implicitly referring to or reminding of linguistic features or structure features”, zu zweiteren „asking pupils to improve language“, „literally repeating correct pupils’ utterances“ und „asking for or explicitly encouraging pupils to independently produce spoken or written language“ (Smit \& van Eerde 2013: 26). In der Tat ließ sich ein solcher Wechsel beobachten, gepaart damit, dass die Schülerinnen und Schüler nach den neun Unterrichtsstunden über ein größeres fachspezifisches Vokabular verfügten. Offen bleibt allerdings das Ausmaß interindividueller Differenzen und somit die Frage, ob alle Schülerinnen und Schüler von dem „whole class scaffolding“ profitierten.

In einem querschnittlich angelegten Design hingegen untersucht KleinschmidtSchinke (2018) Scaffolding-Aktvitäten derselben vier Lehrkräfte in unterschiedlichen Jahrgangsstufen. Ein Fokus sind dabei mikrointeraktionale Stützstrategien, zu denen sie insbesondere implizites und explizites Feedback (negativ und positiv), Expansionen, Reformulierungen, Umformulierungen und „clarification requests“ zählt. Im Vergleich der Jahrgangsstufen zeigt sich, dass Expansionen, die einen Schülerbeitrag nur geringfügig modifizieren, über die Jahrgangsstufen hinweg abnehmen, während Umformulierungen, die in formaler Hinsicht stärker vom 
Schülerbeitrag divergieren, zunehmen. Diese Beobachtung deutet KleinschmidtSchinke dahingehend, dass ältere Schülerinnen und Schüler vermehrt alternative Formulierungen als Input erhalten, die sie selbstständig mit ihrer Ausgangsäußerung abgleichen müssen. Über die Wirksamkeit dieser Art der sprachlichen Unterstützung können aufgrund des querschnittlichen Designs allerdings keine Aussagen getroffen werden. Als zweiten Fokus untersucht Kleinschmidt-Schinke die Adaptivität lehrerseitig etablierter Diskursanforderungen über die Jahrgangsstufen hinweg (2018: 538-548). Zu diesem Zweck werden „Aufgabenstellungen“ kodiert, die die Lehrkräfte im Unterrichtsgespräch an die Schülerinnen und Schüler etablieren, und zwar jeweils nach ihrem Offenheitsgrad und der angezielten kommunikativen Gattung (,Epistemisierungsprofil' bei KleinschmidtSchinke). Es zeigt sich zum einen, dass der Anteil an offenen, komplexere schülerseitige Züge eröffnenden Aufgabenstellungen von der Grundschule bis zur Oberstufe zunimmt (2018: 544). Zum anderen wird in der Grundschule vornehmlich Beschreiben eingefordert ( $\mathrm{u}$ ganz ähnlichen Befunden vgl. Hövelbrinks 2014), während in der Mittelstufe verstärkt Aufgabenstellungen hinzutreten, die auf Erklären und zum Teil auch auf Argumentieren gerichtet sind (vgl. zu entsprechenden Gattungsrepertoires auch Heller, Quasthoff, Prediger et al. 2017; Morek 2016), was sich für die Oberstufe noch einmal verstärkt. Diese Beobachtungen sind vor allem vor dem Hintergrund interessant, dass die genannten Gattungen in ihrer Vertextung und Markierung unterschiedlich anspruchsvoll sind, dass also etwa Beschreibungen als sequenzierende Gattung (Quasthoff 2012) einen weitgehend reihenden, aus der Anschauung entnehmbaren Aufbau ermöglichen, wohingegen Argumentieren eine integrative, kausal bedingte Strukturierung und mithin auch andere sprachliche Formen erfordert (z. B. der Bezugnahme auf Gegenpositionen). Das diskursive Anforderungsniveau scheint also über die Jahrgangsstufen hinweg angepasst zu werden an unterstellbare sprachlich-diskursive Ressourcen ganzer Lernergruppen.

Detaillierteren Aufschluss über Mechanismen des individualisierten Finetuning in Unterrichtsgesprächen liefern Arbeiten, die unmittelbar an Befunde der Diskurserwerbsforschung $\mathrm{zu}$ interaktiven Unterstützungsverfahren in dyadischen Settings anknüpfen und gesprächsanalytisch das sequenzielle Zusammenspiel aus Lehrer- und Schüleräußerungen untersuchen, statt einseitig auf lehrerseitige Verfahren $\mathrm{zu}$ fokussieren (Heller \& Morek 2015). So konnten beispielsweise institutionelle Varianten des Musters Fordern und Unterstützen für Lehrer-Schüler-Interaktionssequenzen in Klassengesprächen der Primar- und Sekundarstufe nachgezeichnet werden. Das Vorkommen entsprechender Verfahren (z. B. Explizieren globaler Zugzwänge, Nachfragen und Rückmeldesignale während der schülerseitigen Produktion eines Äußerungspaketes) - zumindest bei einigen Lehrkräften - liefert erste Evidenzen dafür, dass das in nicht-institutioneller 
Erwachsenen-Kind-Interaktion intuitiv und verständigungsorientiert eingesetzte diskursive Scaffolding durchaus auch für unterrichtliche, d.h. arrangierte und professionelle Lehr-Lern-Interaktionen adaptiert werden kann (vgl. Heller \& Morek 2015: 7). Wenngleich die Wirksamkeit solcher Verfahren noch nicht in der Breite nachgewiesen ist, so sprechen einzelne längsschnittliche Beobachtungen doch dafür, dass die Involvierung in fordernd-unterstützend gestaltete Diskurssequenzen im Unterricht $\mathrm{zu}$ einer Weiterentwicklung der Diskursfähigkeiten der betreffenden Schülerinnen und Schüler führen kann (Heller 2017; vgl. Abschnitt 4.2).

Vereinzelt finden sich überdies Hinweise darauf, dass Lehrkräfte den Grad ihrer kommunikativen Investitionen in das Gelingen schülerseitiger Diskursbeiträge im Austausch mit einzelnen Schülerinnen oder Schülern durchaus vom jeweiligen diskursiven Entwicklungsniveau des/der betreffenden Lernenden abhängig machen (Heller 2012: 234-242; Morek 2012: 223-229). Dass sprachlichdiskursive Anforderungen in situ, d. h. im sequenziellen Zug-um-Zug-Entstehen unterrichtlicher Gesprächssequenzen angepasst werden an schülerseitig gezeigte Kompetenzen, zeigt bspw. Heller (2017): Sie zeichnet nach, wie ein ursprünglicher Zugzwang zum Erklären angesichts schülerseitiger Schwierigkeiten bei dessen Erfüllung in Richtung des sprachlich-diskursiv einfacheren Beschreibens verschoben werden kann. Daneben scheinen jedoch auch andere, nicht erwerbsbezogene Attribuierungen eine Rolle zu spielen (z. B. Attribuierung mangelnden Willens zur Aufmerksamkeit auf Schülerseite; vgl. Heller 2012; Leßmann in diesem Band), die beispielsweise dazu führen können, im Sinne des Matthäus-Effekts nur den ohnehin schon starken Lerner/innen interaktive Unterstützung angedeihen zu lassen.

Zusammenfassend lässt sich demnach festhalten: (1) Zwar wurden Verfahren interaktiver sprachlich-diskursiver Unterstützung im Unterricht beschrieben, doch lag der Fokus vornehmlich auf der Förderung lexikalischer und syntaktischer Ressourcen und seltener auf Fähigkeiten auf Diskursebene. (2) Dass Lehrkräfte im Rahmen diskursiver Praktiken fordernd-unterstützend agieren und dabei globale und lokale Zugzwänge adaptiv einsetzen, konnte zwar vereinzelt gezeigt werden. Dabei wurde jedoch noch nicht systematisch untersucht, ob und mit welchem methodischen Zugang sich ein lehrerseitiges Finetuning in Bezug auf diskursive Kompetenzen individueller Schülerinnen und Schüler feststellen lässt.

Eben in Letzterem besteht aus unserer Sicht ein besonders wichtiges Desiderat: Bislang wurden Verfahren des Mikro-Scaffolding größtenteils kontextentbunden, d.h. ohne ihre jeweilige Einbettung in bestimmte sequenzielle Kontexte beschrieben. Entsprechend werden sie eher als Techniken oder Strategien des Scaffolding ohne Finetuning verstanden, die - zugespitzt formuliert - in unveränderter Form eingesetzt werden können. Aus Sicht der Interaktionalen Diskursanalyse 
sind es aber nicht primär die Strategien an sich, denen eine Erwerbsförderlichkeit zukommt, sondern wesentlich ihrem kontextsensitiven Einsatz: Ob bspw. „Reformulieren“ unterstützend ist, zeigt sich daran, wie ein lehrerseitiger Zug auf etwaige Probleme des vorangegangenen Schülerbeitrags reagiert und wie der oder die Lernende das Scaffolding im anschließenden Zug aufgreift bzw. ,nutzt‘. Erst eine Berücksichtigung solcher sequenziellen Musterhaftigkeiten verspricht genaueren Aufschluss über die lokal-mikrogenetischen sowie ontogenetischen Wirkweisen adaptiver Unterstützung zu geben.

Vor diesem Horizont zentraler Forschungsdesiderata im Bereich lernersensitiver, schulischer Diskurserwerbsförderung zielt dieser Beitrag im weiteren Verlauf darauf, anhand exemplarischer Ausschnitte aus Deutsch- und Mathematikstunden empirisch beobachtbare Realisierungen unterrichtlichen Finetuning sequenziell aufzuzeigen und dessen Konzeptualisierung und Erwerbswirksamkeit theoretisch auszuschärfen.

\section{Empirische Zugänge zum Finetuning}

Forschungsmethodisch ist es nicht trivial, Prozesse des Finetuning von sprachlich-interaktiver Unterstützung empirisch abzubilden, insofern sie immer nur bezogen auf das jeweilige kindliche Erwerbsniveau aufgedeckt werden können. Im Wesentlichen können hier zwei Zugänge gewählt werden:

(1) Es kann erstens ein querschnittlich-interindividueller Vergleich vorgenommen werden. Dabei wird für Kinder unterschiedlichen Alters oder unterschiedlicher Erwerbsstände untersucht, ob und wie erwachsenenseitige diskursive Anforderungen und Unterstützungen in Abhängigkeit von den kindseitig gezeigten Kompetenzen variieren.

(2) Zweitens kann Finetuning über einen mikrolängsschnittlich-intraindividuellen Zugriff untersucht werden. Dabei werden individuelle Leistungsveränderungen zum Maßstab; es wird das erwachsenenseitige Agieren mit einem Kind bzw. einer/einem Lernenden zu unterschiedlichen Zeitpunkten in Bezug gesetzt, um auf diese Weise Anpassungen im Unterstützungsverhalten über die Zeit nachzeichnen zu können.

Im Folgenden sollen beide Herangehensweisen exemplarisch vorgeführt werden, und zwar in qualitativer, gesprächsanalytisch-rekonstruktiver Weise. Wir greifen dabei auf videographierte Deutschstunden in einer ersten Grundschulklasse (DASS-Projekt) sowie Mathematikstunden in einer fünften Klasse der Sekundarstufe (InterPass-Projekt) zurück. Die Transkriptionen folgen dem 
Gesprächsanalytischen Transkriptionssystem 2 (Selting et al. 2009), das im Anhang dieses Bandes einzusehen ist. Analytisch betrachtet werden im Folgenden sowohl explanative (bzw. deskriptive) als auch argumentative Sequenzen. Ziel der Analysen ist es, auszuloten, wie Finetuning im Bereich sprachlich-diskursiver Aneignungsprozesse im Unterricht aussehen und untersucht werden kann. Dabei wird der Blick vor allem darauf gerichtet, in welchen Aufgabenbereichen von Diskurspraktiken (Kontextualisierung, Vertextung, Markierung) Unterstützung zu finden ist, wie sie mit dem kindseitig gezeigten Diskursverhalten zusammenhängt, und wie diskursive Anforderungen verkleinert oder vergrößert werden.

\subsection{Querschnittlich-interindividueller Zugang: Feinabgestimmtes Fordern und Unterstützen diskursiv heterogener Schülerinnen und Schüler in derselben unterrichtlichen Gesprächssituation}

Die Frage, ob und auf welche Weise Lehrkräfte sprachlich-diskursive Anforderungen und Unterstützungsleistungen an verschiedene Lernende anpassen, wird nun in einem ersten Zugriff im querschnittlich-interindividuellen Vergleich des Agierens ein- und derselben Lehrperson mit verschiedenen Lernenden verfolgt. Gegenübergestellt werden Sequenzen aus dem Deutschunterricht einer ersten Klasse, in denen drei Schülerinnen und Schüler mit deutlich unterschiedlichen sprachlich-diskursiven Voraussetzungen jeweils erklärende Äußerungspakete zum Unterrichtsgespräch beitragen (vgl. Morek 2012; s. a. Leßmann in diesem Band). Die Ausschnitte sind einer Stunde entnommen, in der in Vorbereitung eines klasseneigenen Theaterstücks unterschiedliche Puppentheaterformen besprochen werden. In diesem Zusammenhang etabliert die Lehrkraft globale Zugzwänge, die sich auf die Spezifik der jeweiligen Theaterformen bzw. Puppentypen beziehen und insofern schülerseitiges Erklären und/oder Beschreiben erwartbar machen.

Die drei Schülerinnen und Schüler sind TO (Tobias), MI (Michael) und KO (Kornelia); sie tragen nacheinander jeweils unaufgefordert oder auf Nachfrage Erklärungen zu „Schattentheater“ (TO), „Stabpuppen“ (MI) und „Tütenpuppen“ (KO) bei. Für den vorliegenden Beitrag ausgewählt wurden die Drei, weil sich ihre Diskursfähigkeiten in den fraglichen Ausschnitten sehr unterschiedlich darstellen: Während Tobias' Beitrag auf ausgebaute Leistungen in allen drei Dimensionen hinweist (Kontextualisierung, Vertextung, Markierung), zeigt Michael Schwierigkeiten sowohl in der Kontextualisierung als auch in der Vertextung und Markierung; Kornelia wiederum kontextualisiert durchaus aktiv (wenngleich 
nicht ganz passend), hat jedoch noch Schwierigkeiten mit der kohärenten Vertextung ihrer Erklärung.

Die folgende Analyse der drei Beispiele fokussiert die Frage, wie die Lehrkraft ihre Zugzwänge und interaktive Unterstützung in Abhängigkeit von den schülerseitig gelieferten Äußerungen variiert und damit interindividuell unterschiedliche Erwerbsaufgaben fokussiert. Gezeigt wird, wie die Lehrperson in Abhängigkeit von den schülerseitig gezeigten Diskursleistungen entweder so gut wie keine interaktive Unterstützung anbietet (Beispiel 1) oder durch entsprechend adaptierte Zugzwänge und Zuhöreraktivitäten Schülerinnen und Schüler bei der Bewerkstelligung globaler Diskursaufgaben interaktiv unterstützt (Beispiele 2 und 3).

\section{Tobias: Zurückhalten interaktiver Unterstützung bei diskursiver Selbstständigkeit des Schülers}

Betrachten wir zunächst Tobias` Erklärung, die er eigenständig lanciert, nachdem zuvor bereits ,Kasperletheater' und ,Marionettentheater' Gegenstand des Unterrichtsgespräches gewesen sind. Zur besseren Übersicht sind im Transkript die lehrerseitigen Zugzwänge fett gedruckt und die schülerseitigen Äußerungspakete grau hinterlegt.

Beispiel (1): Schattenspiele (TO: Tobias)

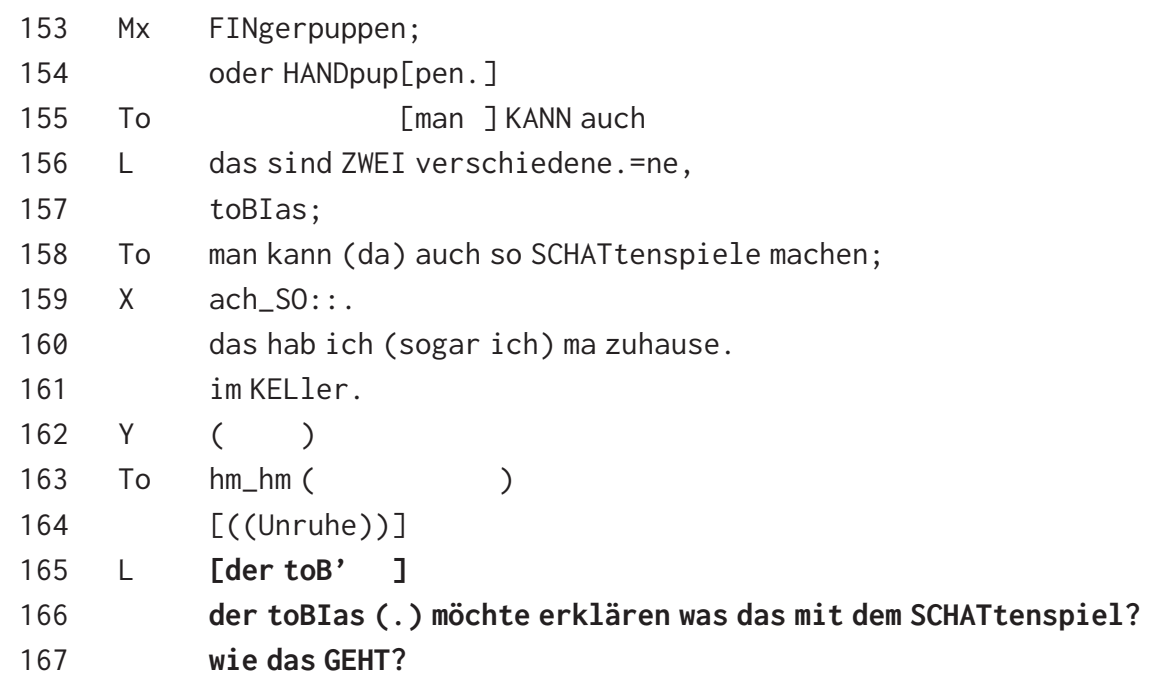


$168 \quad$ ja dann LOS;

169 To hm_da (.)

170 L ((dreht sich z. Tafel, schreibt, Schattenspiel 'an))

171 To ähm da muss man so_ne LAMpe haben?

172 'hh ähm: : man d'=ähm (--) wie so_n grOßes plaKAT,

173 oder irgendwas ANderes oder einfach nur anner WAND,

174 dann kann man sich hinter die äh (. ) vor die LAMpe

175 sowas (.) ähm (. ) ${ }^{\circ} \mathrm{h}$ so FINger zeigen;

$176{ }^{\circ} \mathrm{h}$ also hier äh ähm=ähm SO: : : geht dann das HÄSchen, =

177 =und dann da auch $\mathrm{n}$ SCHMETterling zukommen,

$178 \mathrm{Mi}$ ein WO:LF,

$179 \mathrm{~L}$ das (-) ich KANN das $[()<.<$ cresc $>$ SCHATTenspiel (.)

so wie TOB' ]

$180 \mathrm{X} \quad$ [ja schattenspiel geht SO: ( )]

$181 \mathrm{~L} \quad$ [so wie toBIas gerade gesagt] hat

$182 \times \quad[(\quad)]$

$183 \mathrm{~L}$ tatsächlich mit den> (.) ! FIN! gern machen,

184 es GEHT aber auch ANders;

$185 \mathrm{Y} \quad(\quad[\quad)]$

186 L [ [hat $)]$ schomma jemand ein ANderes

$187 \mathrm{~L} \quad$ [ein ANderes schattenspiel?]

188 To [ja_a (.) man kann auch PUP' ]

189 To <<all> ähm=ähm PUPpen nehmen;>

190 und se dann daVOR halten.

191 ja_a;

192 L 0::der man kann sich auch fiGU: ren ausschneiden,

193 und die figuren führen.=ne?=

$194 \quad Z \quad=j a$

$195 \mathrm{~L}$ das is AUCH das geht auch beim SCHATtenspiel.

$196 \times \quad[<<p>(\quad)>]$

$197 \mathrm{~L} \quad$ [FÄLLT jemand ]

198 L fällt jemandem f'noch ein !AN! deres (-) theAter (--) ein?

Mit seinem Zwischenruf „,man KANN auch [. . .] man kann (da) auch so SCHATtenspiele machen;“ (Z. 155 und 158) thematisiert TO initiativ eine weitere Theaterform. Damit bietet er sich implizit als deren möglicher Erklärer an. Auf genau diese Bereitschaft fokussiert die Lehrerin entsprechend, indem sie ihm sehr explizit das Rederecht für ein erklärendes Äußerungspaket zuweist („der toBIas (.) möchte erklären was das mit dem SCHATtenspiel? wie das GEHT?, 
Z. 165-167; „ja dann LOS;“, Z. 168). Dass TO in der Lage ist, eine entsprechende Erklärung zu liefern, wird stillschweigend vorausgesetzt; die zum Ausdruck gebrachte lehrerseitige Attribuierung bezieht sich lediglich auf die Frage des ,Wollens' (,möchte erklären'), wie es etwa auch von Hausendorf \& Quasthoff (1996: 213f.) als typische Haltung von erwachsenen Zuhörern gegenüber 7-Jährigen beobachtet werden konnte.

Tatsächlich liefert TO sodann selbstständig und ohne jede Hilfe eine ausgebaute Erklärung in Form eines komplexen Äußerungspaketes (Z. 171-177). Sowohl Vertextung als auch Markierung folgen gattungsspezifischen Erwartbarkeiten: Nach der Einführung des benötigten Materials (Z. 171-173) und der Thematisierung des Aufbaus (Z. 174) wird das „Finger-Zeigen“ als zentrale Handlung beim Schattentheater benannt (Z. 175), die durch zwei Beispiele - „Häschen“ und „Schmetterling“ - sowohl verbal als auch gestisch veranschaulicht wird (Z. 176-177); dazu wählt TO sprachliche Formen wie z. B. das unpersönliche Pronomen man, die Modalverben können und müssen oder Konstruktionen des Vergleichens (,wie so ein“, Z. 172) und exemplarischen Zeigens („,so geht dann“, Z. 176), um sein Äußerungspaket als Erklärung zu markieren.

Entsprechend können unterstützende Zuhöreraktivitäten seitens der Lehrerin ausbleiben - ja, sie kann sogar parallel zu TOs Redebeitrag einen Tafelanschrieb anfertigen (Z. 170) und TO so die Verantwortung für das Gelingen der Erklärung vollkommen selbst überlassen. Vor diesem Hintergrund muss auch die verfrühte Rückübernahme des Rederechts (Z. 179f.) durch die Lehrerin gedeutet werden: Mit einem metasprachlichen, retrospektiven Verweis auf TOs Beitrag, der an die ganze Klasse adressiert ist (,so wie toBlas gerade gesagt hat“, Z. 181) behandelt sie seine Erklärung als inhaltlich vollständig, nachvollziehbar und für alle übrigen Schülerinnen und Schüler zur inhaltlichen Weiterarbeit („es GEHT aber auch anders“, Z. 184) verfügbar. Dass TO trotzdem und in proaktiver Vervollständigung der lehrerseitigen Äußerung die Erklärung fortsetzt (,ähm = ähm PUPpen nehmen; und de dann daVOR halten.“, Z. 189f.), unterstreicht einmal mehr seine diskursiven Fähigkeiten v.a. im Kontextualisieren und Vertexten. Letztlich schließen TO und die Lehrerin die Schattentheater-Erklärung auf kollaborative, einander wechselseitig ergänzende Weise ab (Z. 188-195).

\section{Michael: Verringern der Anforderungen und umfassende Unterstützung}

Ganz anders gestaltet sich die Lehrer-Schüler-Interaktion im Falle Michaels. Er muss überhaupt erst von der Lehrperson zur Beteiligung am Unterrichtsgespräch eingeladen werden und hat Schwierigkeiten, selbstständig eine angemessene Erklärung oder Beschreibung zu liefern. Die folgende Sequenz zeigt, 
wie es der Lehrerin durch das feinabgestimmte Setzen von Zugzwängen und die fortwährende Aufrechterhaltung einer dyadischen Sequenz mit ihm gelingt, eine Beschreibung von Michael zu elizitieren und ihn bei deren Vollzug zu unterstützen.

Nachdem die Lehrerin zuvor danach gefragt hat, ob ,jemandem f'noch ein !AN!deres (-) theAter (-) ein[fällt]“ (Z. 198, s. Beispiel 1), wendet sie sich wie folgt an MI (Z. 229 und 233), ohne dass dieser sich gemeldet hat:

Beispiel (2): Stabpuppen (MI: Michael)

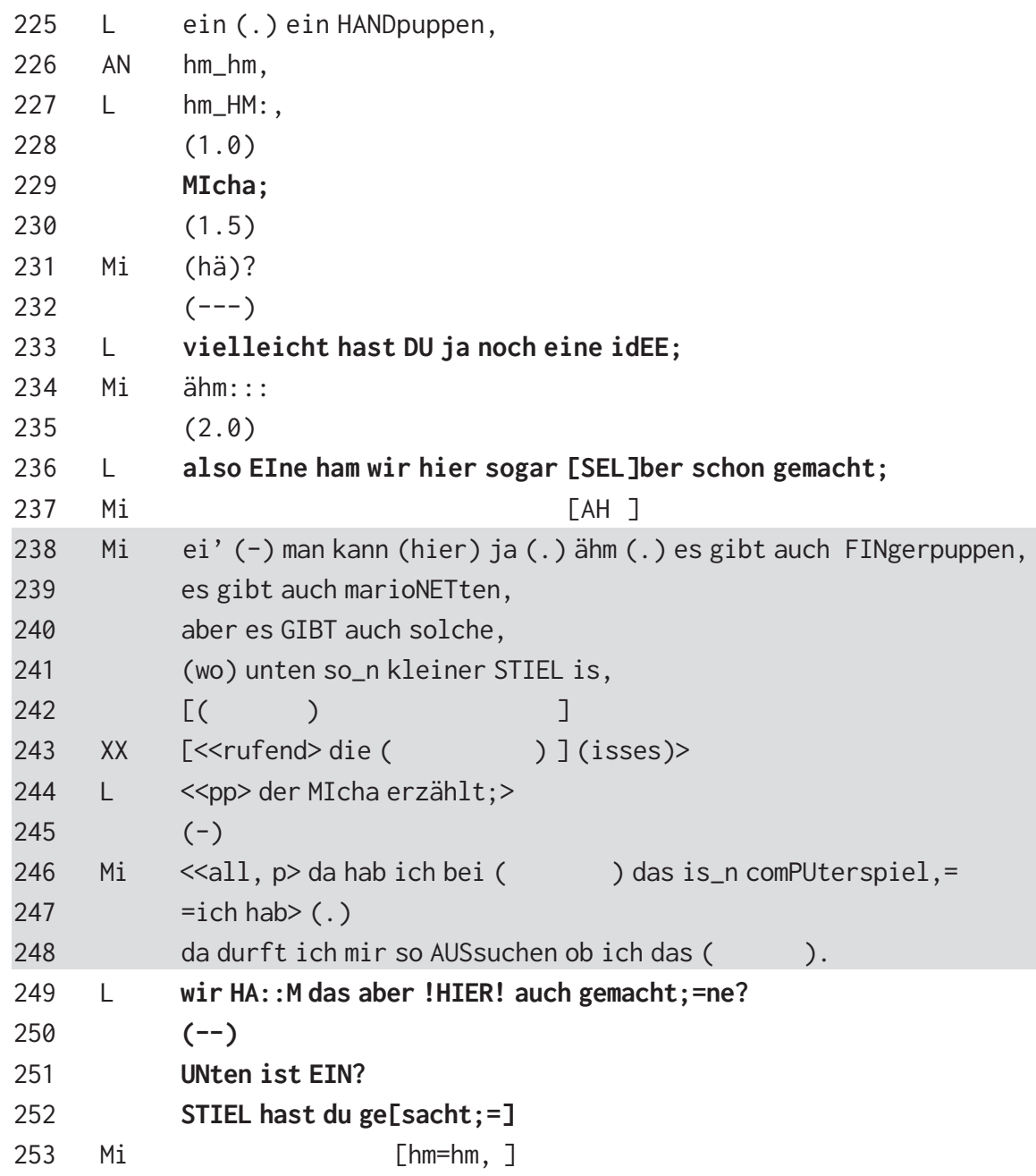




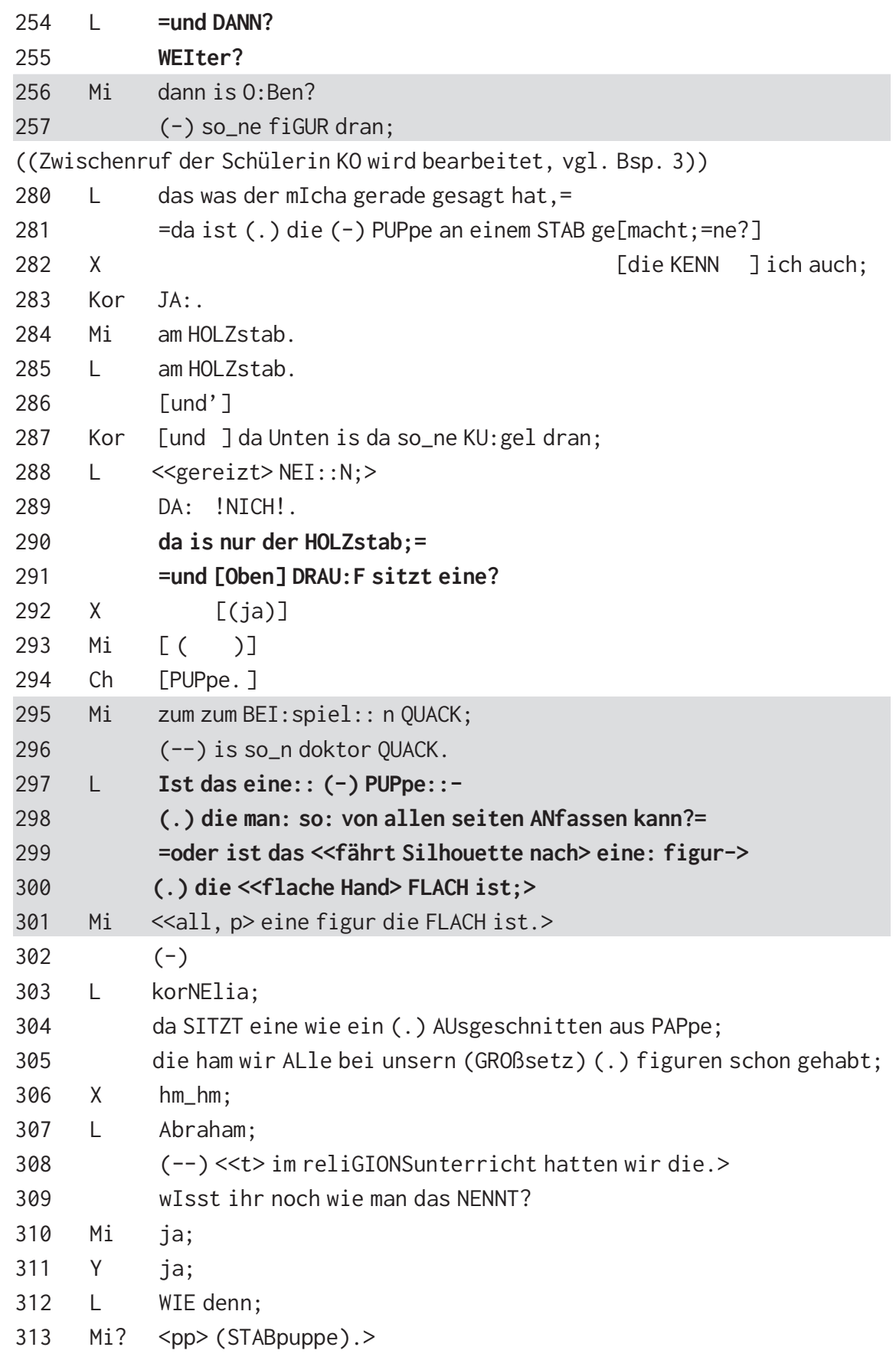




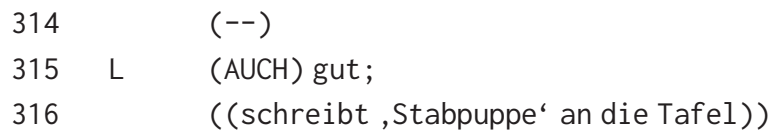

Anders als zuvor TO bemüht sich MI nicht um den Erhalt des Rederechts. Das namentliche Aufrufen (Z. 229) orientiert MIs Aufmerksamkeit überhaupt erst einmal auf den Unterrichtsdiskurs, bevor er mit der Folgeäußerung „vielleicht hast DU ja noch eine idEE;“ (Z. 233) als prospektiver Beiträger zum Unterrichtsgespräch involviert wird. Sowohl die abschwächende Modalisierung (vielleicht) als auch die syntaktische Form (Aussageformat statt etwa Frage) tragen dazu bei, die interaktive Verpflichtung für MI und das kommunikative Anforderungsniveau gering zu halten: Etabliert wird die Anschlusserwartung, einen weiteren, bisher nicht behandelten Theatertyp zu benennen; diesen näher zu charakterisieren, wäre in Reaktion auf diese Lehreräußerung zwar möglich, jedoch nicht unbedingt nötig. Mindestens jedoch muss ein passender Referent gefunden und thematisiert werden, was MI allerdings nicht auf Anhieb gelingt, wie sein Zögern dokumentiert (Z. 234-235).

Bemerkenswert ist nun - gerade vor dem Hintergrund des mehrfach beschriebenen Musters, das Rederecht einfach an nächste Schüler/innen weiterzuvergeben (vgl. Böttcher 1994; Heller 2012: 221f.) -, dass die Lehrerin weiterhin MI adressiert. Mit ihrer Äußerung „also EIne ham wir hier sogar SELber schon gemacht;“ (Z. 236) gibt sie ihm eine Hilfestellung in Form eines „Winks“ (Ehlich \& Rehbein 1986: 27) für die Suche einer möglichen Antwort: Sie verweist auf das gemeinsame Basteln einer bestimmten Puppenart im vergangenen Unterricht, was schließlich entsprechende Erinnerungen bei MI aufruft (Z. 237-239). In Abgrenzung zu den zuvor bereits besprochenen Puppen thematisiert er „solche, (wo) unten so_n kleiner STIEL is,“ “(Z. 240f.). Er liefert hier also tatsächlich eine erste beschreibende Information zur äußeren Beschaffenheit des nun fokussierten Puppentyps. ${ }^{2}$ Die helfende Zuhörerreaktion der Lehrperson resultiert in einer erfolgreichen Involvierung Michaels.

Im weiteren Ausbau seines Beitrags zu einem tatsächlichen Äußerungspaket allerdings driftet MI sowohl in Inhalt als auch in der Gattung ab (,das is_n computerspiel, = ich hab (.) da durft ich mir so AUSsuchen ob ich das ( )“, Z. 246-248): Sein Beitrag verschiebt sich hin zu einer narrativen Wiedergabe eines Erlebnisses mit einem Computerspiel.

2 Aufgrund von Unruhe in der Klasse sind Michaels Folgeäußerungen (Z. 242, 246) nicht bzw. nur unvollständig zu verstehen; die Lehrerin interveniert (Z. 244). 
Trotzdem entlässt ihn die Lehrperson nach wie vor nicht aus der Bearbeitung der globalen Anforderung. ${ }^{3}$ Vielmehr richtet sie verschiedene Zuhöreraktivitäten an ihn, die die Aufgabe des Beschreibens für MI aufrechterhalten und Hilfestellung bei deren Bearbeitung geben. Als erstes re-orientiert sie MIs Aufmerksamkeit auf die eingangs von ihm thematisierten Puppen, und zwar mit einer refokussierenden Vergewisserungsfrage (,wir HA::M das aber !HIER! auch gemacht;= ne?“, Z. 249); die danach gelassene Pause (Z. 250) böte MI Gelegenheit, seinen Redebeitrag wiederaufzunehmen und auszubauen. Als dies ausbleibt, reformuliert die Lehrerin MIs ursprüngliche Äußerung (,UNten ist EIN? STIEL hast du gesacht;“ Z. 251-252) und stellt sie ihm zur Ratifikation und potenziellen Fortsetzung anheim. Damit setzt sie einen impliziten Zugzwang zur Elaborierung der begonnenen Beschreibung. Als MI diesen (noch) nicht bedient (vgl. lediglich sein Bestätigungssignal, Z. 253), wählt die Lehrerin schließlich mit Fortsetzungsfragen (,und DANN?“, Z. 254; „WEIter?“, Z. 255) ein sehr stark steuerndes zuhörerseitiges Mittel des Einklagens von Elaborierungen. Zugleich wird die globale Anforderung dadurch lokalisiert, insofern nun auch eine rein satzförmige Fortsetzung durch MI möglich wird. Durch die Hervorhebung des Konnektors und DANN (Z. 254) bietet sie MI zudem auch auf Markierungsebene Hilfe für die gattungsspezifische Vertextung von Gegenstandsbeschreibungen, nämlich auf das von einem Anfangspunkt startende linear-reihende Voranschreiten. Insgesamt also werden die diskursiven Anforderungen an MI sukzessive in ihrer Höhe dem von MI Geleisteten und offenbar Leistbaren angepasst.

Tatsächlich kommt MI nach den Fortsetzungsfragen dem Zugzwang zur deskriptiven Elaborierung nach („dann is O:Ben? (-) so_ne fiGUR dran;“, Z. 256-257).

Nach einer mitschülerseitigen Intervention lenkt die Lehrerin zurück in die Beschreibung (Z. 280). Durch eine mitkonstruierende Zuhöreraktivität in Form einer „designedly incomplete utterance“ (Lerner 1995) erleichtert sie Mi den Wiedereinstieg in seine Beschreibung (,und Oben DRAU:F sitzt eine?“, Z. 291): MI braucht die begonnene Äußerung nur noch zu vervollständigen (vermutlich Z. 293). Zur Verkleinerung der diskursiven Anforderungen trägt hier auch bei, dass die Lehrerin in ihrer Zuhörerfrage sowohl den nächsten Ansatzpunkt für die Vertextung („oben drauf“) als auch für die Markierung (Formulierungsmöglichkeit „sitzt“) anbietet.

3 Beispielsweise könnte die Lehrerin MI auch explizit auf die mangelnde Passung seines Beitrags hinweisen (etwa: ,Wir erzählen hier gerade aber nicht von Computerspielen') oder ihn aus der Rolle des primären Sprechers entlassen (vgl. dazu z. B. Heller 2012: 221f.). 
Bemerkenswert ist allerdings, dass MI nicht bei einer bloßen Äußerungsvervollständigung stehenbleibt. Vielmehr baut er seine Vertextung mit einem Beispiel aus und wählt damit ein gattungsspezifisches Mittel, das der Veranschaulichung dient (,zum zum BEI:spiel:: n QUACK;“, Z. 295).

Auch danach hält die Lehrerin den Zugzwang zur Fortsetzung und Präzisierung der Beschreibung noch weiter aufrecht: Mit einer mitkonstruierenden Zuhöreraktivität in Form einer Alternativfrage bringt sie aus der Zuhörerrolle das zu beschreibende Merkmal ,Dimensionalität‘ ein („Ist das eine:: (-) PUPpe::- (.) die man: so: von allen seiten ANfassen kann?“, Z. $297 \mathrm{f} . ;$ „oder ist das eine: figur- (.) die FLACH ist;“, Z. 299-300). Wieder werden sprachlich-diskursive Anforderungen für MI reduziert: Die beiden mit oder verbundenen Entscheidungsfragen etablieren für MI nunmehr einen lokalen Zugzwang und sie enthalten bereits dasjenige sprachliche Material (hier: spezifizierende Relativsätze), das bei der Bewerkstelligung des fraglichen Vertextungsschrittes genutzt werden kann. Folglich braucht MI ,nur die inhaltlich passende der beiden lehrerseitig zur Verfügung gestellten Formulierungen zu wiederholen („eine figur die FLACH ist.“, Z. 301).

Insgesamt zeigt diese zweite Sequenz, dass und wie die Lehrperson in der bilateralen Interaktion mit einem diskursiv schwächeren Schüler die diskursiven Anforderungen zur Bewerkstelligung eines globalen Zugzwangs (hier: zum Beschreiben) konsequent anpasst: Zunächst wird die Hürde zum Eintritt in eine deskriptive Diskurseinheit gesenkt. Nach erfolgter Übernahme der primären Sprecherrolle und manifest werdender Schwierigkeiten bei der Vertextung werden die Anforderungen angehoben und vereindeutigt, bevor dann die lehrerseitige Unterstützung gegen Ende der Beschreibung stärker steuernd und mitkonstruierend ausfällt. Michaels Beiträge erfahren dabei systematisch eine Als-ob-Behandlung: Obwohl sie für sich genommen noch keine hinreichende Beschreibung liefern, nutzt die Lehrperson sie fortwährend als Teilschritte im Rahmen einer gemeinsamen Verständigungssicherung darüber, wie denn die fragliche Puppe aussieht. Auf diese Weise gelingt es der Lehrerin, einen zuvor nicht partizipierenden Schüler in den deskriptiv und explanativ orientierten Unterrichtsdiskurs zu involvieren und ihm sprachlich-interaktive Hilfestellungen anzubieten. Letztlich vermag somit MI trotz seiner eingeschränkteren diskursiven Fähigkeiten eine ausgebaute, inhaltlich vollständige und kohärente Beschreibung der „Stabpuppen“ (Z. 313-316) beizutragen. 


\section{Kornelia: Austarieren diskursiver Unterstützung bei Diskrepanz zwischen Selbstständigkeit und inhaltlicher Passung}

Betrachten wir nun einen dritten Fall aus derselben Unterrichtsstunde, der gewissermaßen ,zwischen' den Beispielen (1) und (2) verortet ist: Wie zuvor TO bemüht sich KO initiativ um das Rederecht, um einen erklärenden Beitrag zu einem Puppentheater zu liefern. Während sie also einerseits aktiv kontextualisiert, offenbart ihr Beitrag andererseits, dass sie ähnliche Schwierigkeiten wie MI hat, eine kohärente Diskurseinheit selbstständig zu vertexten. Im Sinne eines feinabgestimmten Forderns und Unterstützens setzt die Lehrperson in Reaktion darauf ganz ähnliche Verfahren des Verkleinerns von Anforderungen ein wie bei MI; allerdings fokussieren diese primär die Ebene der Vertextung, da KO beim Kontextualisieren und Markieren bereits selbstständiger agieren kann. Insgesamt werden somit in sequenzieller Abstimmung mit dem schülerseitig Gezeigten etwas höhere sprachlich-diskursive Anforderungen an KO etabliert.

Beispiel (3): Tütenpuppen (Ko: Kornelia)

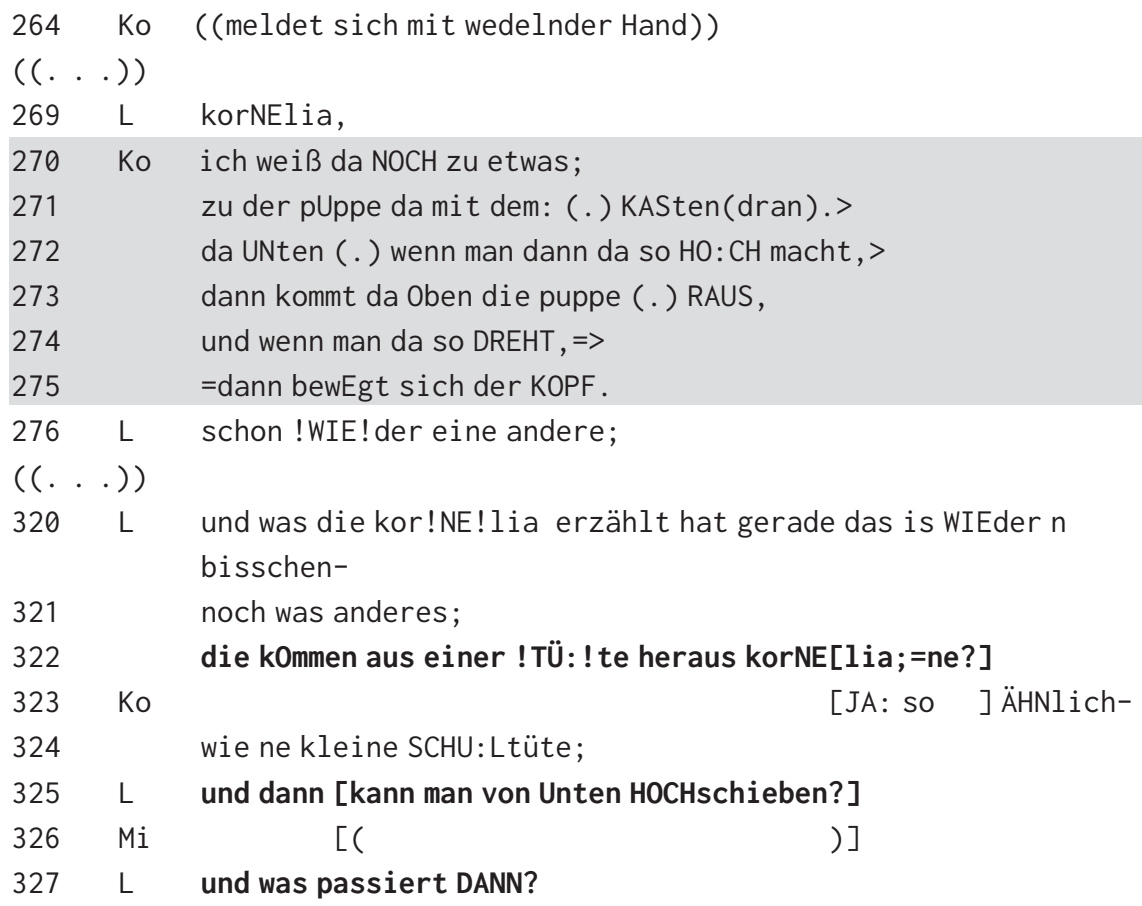




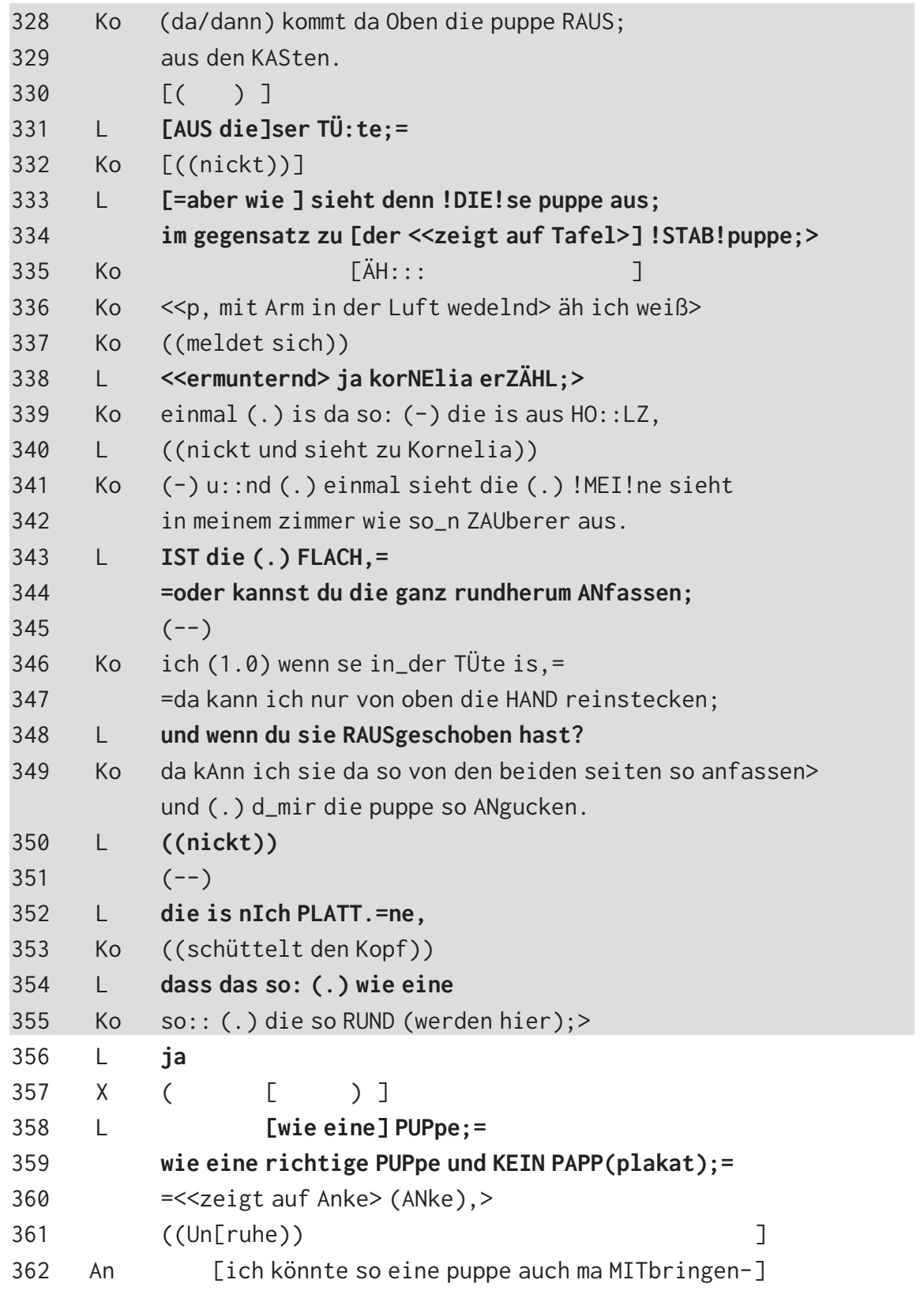


Auf eigene Initiative hin (,ich weiß da NOCH zu etwas;“ Z. 270) liefert KO - nach Erhalt des Rederechts durch die Lehrerin (Z. 269) - selbstständig ein Äußerungspaket erklärenden Typs (Erklären-Wie): Mit für Spielerklärungen typischen wenndann-Gefügen expliziert sie die Zusammenhänge zwischen Spieler- und Puppenbewegungen, d. h. sie erklärt, wie eine bestimmte Puppenart durch eine Aufwärtsbewegung aus einem Behältnis geschoben und ihr Kopf in Bewegung versetzt werden kann (Z. 271-275). Wenngleich ihr Beitrag an der Stelle im Gespräch zunächst als nicht passend zurückgewiesen wird (,schon !WIE!der eine andere;“, Z. 276), so zeigt er doch, dass KO durchaus erkannt hat, dass Äußerungspakete erklärenden Typs von Schülerseite gerade gefragt sind. Dass KO aber eingangs weder benennt noch beschreibt, auf welche Art von Puppenform sie genau referiert, erschwert die inhaltliche Einordnung des Beitrags.

Später gewährt die Lehrerin KO mittels einer refokussierenden Vergewisserungsfrage das Rederecht, ihr Äußerungspaket wiederaufzunehmen (,die kOmmen aus einer !TÜ:!te heraus korNElia;=ne?“, Z. 322). ${ }^{4}$ Dieser qua lokalem Zugzwang zunächst verkleinerten diskursiven Anforderung kommt KO nach, indem sie eine illustrierend-vergleichende Präzisierung liefert (,JA: so ÄHNlich wie ne kleine SCHU:Ltüte;“ Z. 323f.). In ein global organisiertes Äußerungspaket steigt sie indes nicht (wieder) ein.

In Reaktion darauf steuert die Lehrerin nun ganz ähnlich in ein Äußerungspaket, wie sie das auch in der Sequenz mit MI getan hat: Sie legt erst eine refokussierende Reformulierung in Funktion einer Rückfrage nach (,und dann kann man von Unten HOCHschieben?“, Z. 325), dann formuliert sie eine explizite Fortsetzungsfrage (,und was passiert DANN?“, Z. 327). Damit liefert sie auch KO interaktive Hilfestellungen für die (erneute) Vertextung, die jeweils Startpunkt, Richtung und reihendes Muster der Vertextung vorgeben.

Nach einer weiteren Verständnissicherungsschleife zur Bezeichnung „Tüte“ (Z. 331-332) setzt die Lehrerin anschließend einen eindeutig deskriptiv ausgerichteten globalen Zugzwang zum Aussehen der Tütenpuppe (Z. 333-334: ,aber wie sieht denn !DIE!se puppe aus;“). Damit fokussiert sie nun genau den inhaltlichen Part, der KOs Ursprungsbeitrag gefehlt hatte. Gerichtet ist der Zugzwang allerdings nicht unmittelbar an KO, sondern an die gesamte Klasse. Auch bringt er erhöhte sprachlich-diskursive Anforderungen mit sich: Es muss aus der wieFrage geschlossen werden, dass nicht bloß eine einzelne Äußerung, sondern ein Äußerungspaket anzuschließen ist - und es wird darüber hinaus eine kontrastie-

4 Inhaltlich wird dabei das Ursprungslexem Kasten durch das Lexem Tüte ersetzt, d. h. es findet eine lexikalische Ersetzung auf Ebene der Markierung statt. 
rende Vertextung projiziert (,im gegensatz zu der <<zeigt auf Tafel > !STAB! puppe;“, Z. 334).

Als KO nun erneut aktiv kontextualisiert und sich sehr deutlich um den (Rück)Erhalt des Rederechts bemüht (Z. 335-337), weist die Lehrerin ihr mit der formalen Explikation eines globalen Zugzwangs die primäre Sprecherrolle wieder zu (,ja korNElia erZÄHL;“, Z. 338). Es offenbart sich jedoch, dass KO entgegen ihrer angezeigten Bereitschaft nicht in der Lage ist, ohne Hilfe eine kohärente Beschreibung zu liefern. Zwar ist sie um eine tatsächlich kontrastive Vertextung bemüht („einmal (.) is da so: [. . .] u::nd (.) einmal sieht die“, Z. 339 und 341), doch driftet sie - wie auch MI in Beispiel 2 - schließlich in die Wiedergabe subjektiven Erfahrungswissens ab (,!MEI!ne sieht in meinem zimmer wie son ZAUberer aus.“, Z. 341-342).

In Reaktion auf diese manifest werdenden Vertextungsschwierigkeiten greift die Lehrerin sodann auf ähnliche, wenngleich weniger stark mitkonstruierende Unterstützungsverfahren zurück wie in der Sequenz mit MI (Beispiel 2): Auch KO wird nicht aus der Bearbeitung der globalen Anforderungen entlassen; teils wortgleich stellt die Lehrerin KO eine Alternativfrage, die sich auf die Dimensionalität der zu beschreibenden Puppe bezieht („IST die (.) FLACH, = oder kannst du die ganz rundherum ANfassen;“, Z. 343-344), und eine mitkonstruierende Elaborierungsfrage (,und wenn du sie RAUSgeschoben hast?“, Z. 348). Anders als MI reichert KO bei der Beantwortung der Zuhörerfragen die lehrerseitigen Formulierungen allerdings um eigene Präzisierungen an, beispielsweise ersetzt sie die lehrerseitig verwendete Adverbialphrase „ganz rundherum“ durch die Präpositionalphrase „von beiden seiten“ (Z. 349) und liefert eine inhaltliche und formale Vervollständigung des subjektiv entstehenden Sichteindrucks (,dass das so: (.) wie eine“ - KO: „so:: (.) die so RUND (werden hier);“, Z. 354).

Insgesamt kommt es im Zusammenspiel lehrerseitiger lokaler Zugzwänge und entsprechender Antworten von KO zu einem kollaborativen Abschließen zwischen Lehrerin und Schülerin - ähnlich, wie es auch bei TO und MI der Fall war. Im interaktiven Zusammenspiel aus Schüler- und Lehreräußerungen werden so die diskursiven Anforderungen fortwährend in ihrer Höhe justiert, wobei KO v. a. im Bereich der Vertextung Unterstützung erhält.

Insgesamt zeigt der Vergleich des Agierens der Lehrperson in den exemplarischen Sequenzen mit drei unterschiedlichen Lernenden, dass und wie die Lehrperson die Setzung und Wiedereinsetzung globaler Zugzwänge sowie deren sequenziell gestaffelte Modifikation und Verkleinerung in Abhängigkeit der Lernenden und ihrer Interaktionsbeiträge vornimmt. Tabelle 1 fasst die Befunde überblicksartig zusammen: 


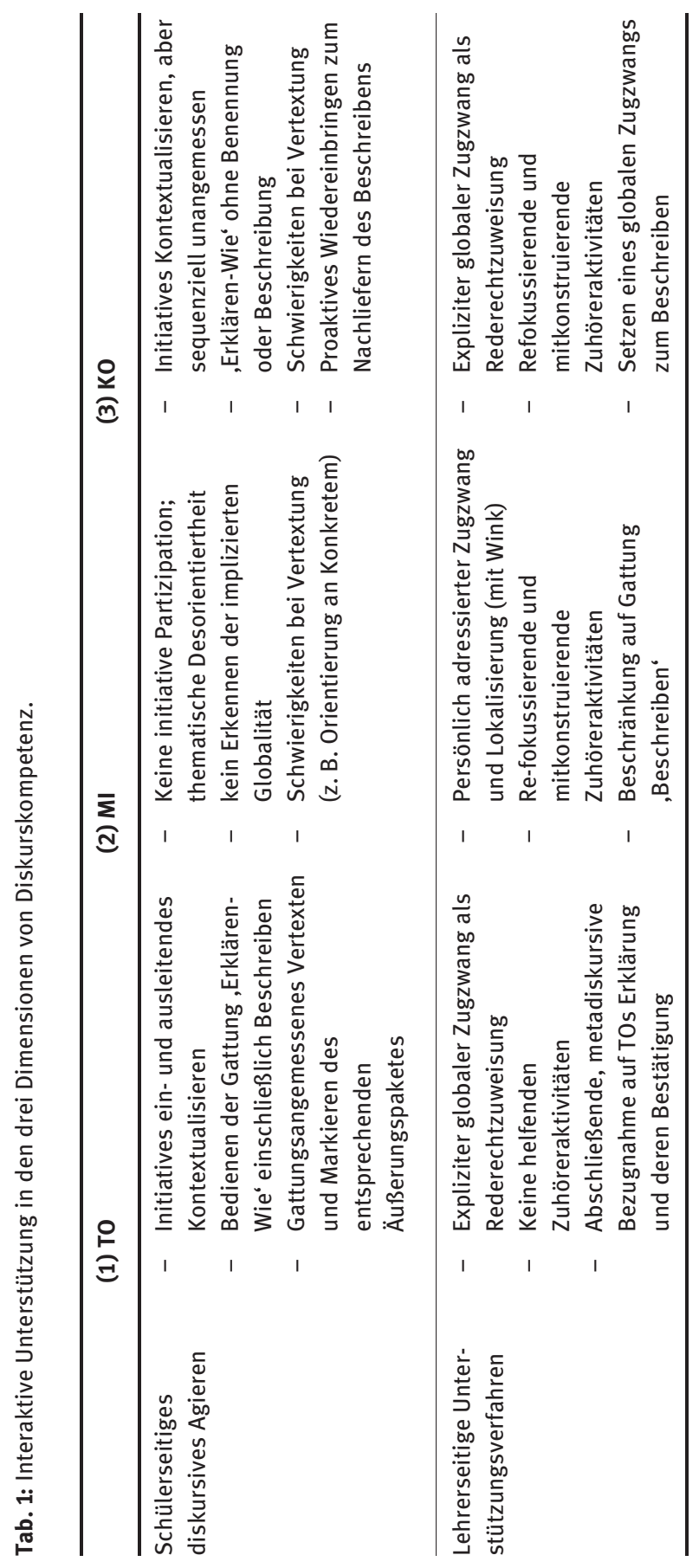


TO agiert auf allen drei Ebenen diskursiver Aufgaben (Kontextualisierung, Vertextung, Markierung) eigenständig, situations- und gattungsangemessen; folglich bedarf es in der lehrerseitigen Interaktion mit ihm keinerlei Verringerung sprachlich-diskursiver Anforderungen oder interaktiver Unterstützung. Anders bei MI und KO: Ihr Agieren in den ausgewählten Unterrichtssequenzen lässt Schwierigkeiten in allen Dimensionen von Diskurskompetenz erkennen (MI) bzw. - trotz hohem Partizipationsbemühen und aktiver Kontextualisierung - Schwierigkeiten im Bereich von sequenzieller Passung und gattungsangemessener, kohärenter Vertextung (KO). Statt in diesen Fällen die betreffenden Schülerinnen und Schüler gar nicht erst zu involvieren oder das Rederecht nach dem nicht angemessenen Bedienen von Zugzwängen an Mitschüler/innen weiterzugeben, reagiert die Lehrerin jeweils aus der Zuhörerrolle gemäß des Musters Fordern und Unterstützen. Dabei setzt sie verschiedene Verfahren ein, z. B. das Lokalisieren von Zugzwängen, das Anbieten helfender Zuhöreraktivitäten und das selektive Fokussieren bestimmter Gattungen. Diese Verfahren werden - wie die sequenzanalytische Rekonstruktion offengelegt hat - keineswegs schematisch eingesetzt, sondern sind jeweils feinabgestimmt auf die Diskursbeiträge und das damit jeweils gezeigte inhaltliche und sprachlich-diskursive Vermögen der beteiligten Schüler/innen. So etwa erhält MI, der zunächst kein Äußerungspaket anschließt und später vom Beschreiben in ein assoziatives Erzählen verfällt, starke interaktive Unterstützung, die die diskursiven Anforderungen für ihn auf ein bearbeitbares Maß reduziert.

Gerade der dritte vorgestellte Fall KOs zeigt allerdings auch, dass das angemessene Justieren lehrerseitiger Zugzwänge $u$. U. auch nach dem trial-anderror-Verfahren verläuft: So etwa muss die Lehrkraft nach der wechselseitigen Etablierung eines globalen Zugzwangs im Verlauf von dessen Bearbeitung Anforderungen wieder verkleinern, als sich Schwierigkeiten im Verlauf des Vertextens offenbaren. Insofern bedeutet Finetuning in Unterrichtsgesprächen wohl mehr noch als in der dyadischen Eltern-Kind-Interaktion -, dass sehr genaues, sequenziell mitprozessierendes Zuhören eine wichtige Voraussetzung ist, um für jede Schüleräußerung online entscheiden zu können, welche Zuhöreraktivitäten dem Schüler oder der Schülerin an der betreffenden Stelle seines oder ihres Diskursbeitrages beim Bedienen globaler Diskursanforderungen helfen.

\subsection{Mikrolängsschnittlich-intraindividueller Zugang: Feinabgestimmtes Fordern und Unterstützen desselben Schülers über die Zeit}

Der folgende Abschnitt demonstriert einen mikrolängsschnittlich-intraindividuellen Zugang zu feinabgestimmtem Fordern und Unterstützen, indem Interaktionen 
eines Lehrers mit ein- und demselben Schüler (Pekcan) in zeitlich aufeinanderfolgenden, aber vergleichbaren Situationen untersucht werden. Betrachtet werden Auszüge aus ähnlich gelagerten Argumentationen, die aus zwei Mathematikstunden der fünften Jahrgangsstufe stammen, in denen Pekcan vor die Anforderung gestellt ist, globale Zugzwänge zum Argumentieren autonom zu erkennen und zu bedienen. Die Analyse rekonstruiert zum einen, wie die interaktive Anforderung und Unterstützung in der ersten Unterrichtsstunde auf das von dem Schüler gezeigte Kompetenzniveau im Bereich der Kontextualisierung abgestimmt wird; dies zeigt sich im Vergleich zweier Sequenzen aus demselben Unterrichtsgespräch, in denen Pekcan jeweils gefordert ist, seine Position zu begründen. Zum anderen wird nachgezeichnet, wie die diskursive Anforderung in der darauffolgenden Stunde angehoben wird. In dieser dritten Sequenz wird deutlich, dass Pekcan in genau dem Bereich selbstständiger agiert, auf den in der ersten Sequenz die lehrerseitige Unterstützung zielte: die Kontextualisierung globaler Zugzwänge zum Argumentieren.

Anforderungsniveaus im Bereich der Kontextualisierung unterscheiden sich in Abhängigkeit davon, ob globale Zugzwänge (vgl. 2.2) in expliziter oder impliziter Form etabliert werden. Explizite Zugzwänge beinhalten eine metadiskursive Benennung der Gattung, z. B. ,Sag mir einen Grund, warum . . . Implizite Zugzwänge können entweder mittels einer gattungsindizierenden Frage (,Warum denn?`) gesetzt werden; es kann aber auch gänzlich dem Rezipienten überlassen sein, eine konditionelle Relevanz aus dem sequenziellen Kontext zu schließen, wenn bspw. bereits ein Dissens etabliert ist oder der Vorredner bereits eine Position begründet hat. Die Erwerbsforschung zeigt, dass Kinder zunächst explizite globale Zugzwänge $\mathrm{zu}$ bedienen lernen, bevor sie auch implizite oder aus dem Kontext zu erschließende konditionelle Relevanzen erkennen können (Hausendorf \& Quasthoff 2005).

Den mathematischen Gegenstand der hier untersuchten Argumentationen bildet die Frage, wie man Gruppen mit Blick auf bestimmte Parameter, bspw. die Größe, vergleichen kann. Diese Frage wird auf die Klasse angewendet, um auf diese Weise induktiv die Funktion des Durchschnitts zu erarbeiten.

\section{Pekcan I: Impliziter globaler Zugzwang zum Argumentieren mit gattungsindizierender Frage}

Konkret setzt sich die Klasse mit folgender Frage auseinander: Welche Gruppe hat die größeren Füße - die der Mädchen oder die der Jungen? Dazu haben die 19 Mädchen der Klasse jeweils einen Schuh ausgezogen und ihre Schuhe aneinandergereiht; die 12 Jungen sind ebenso verfahren. Erwartungsgemäß ist die Schuhreihe 
der Mädchen länger. Mit seiner Frage danach, wer denn jetzt „ge!WON!nen“ hat (Z. 2), provoziert der Mathematiklehrer eine Flut von spontan in den Raum gerufenen Positionsbekundungen.

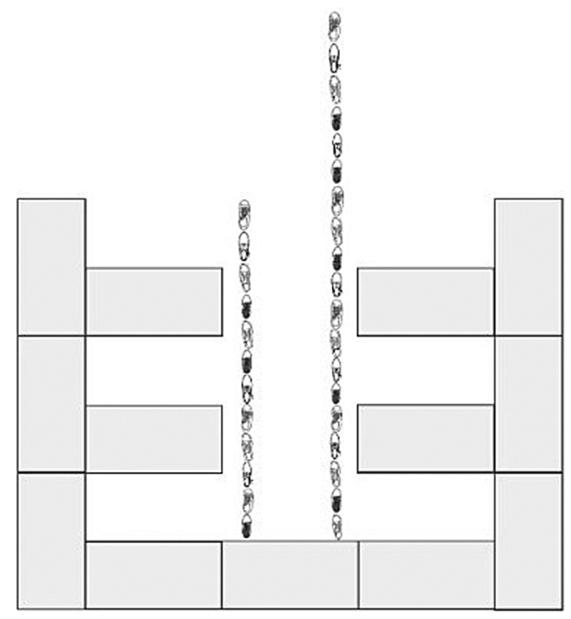

Abb. 1: Schuhreihen zwischen den Schülertischen.

Beispiel (4) 01-5-MU-07-22492909_Wer hat denn jetzt gewonnen_1

001 leh jetzt hab_ich schon ganz grade: : (-) ganz viele kommenTAre dazu gehört;

002 wer hat denn jetzt ge!WON!nen;

003 pek (-) WIR;

004 sus WIR;

005 un1 ja die sind ja auch MEHR;

006 pek ja die sind doch MEHR, (.)

007 das ist Unfair;

008 sus ((rufen spontan Positionen in den Raum))

009 leh SIlas;

010 sil das GEgenteil von den jungen hat gewonnen;

011 leh (-) wer hat [gew-]

012 un2 [die ] MÄDchen;

013 leh (---) das ge (.) die MÄDchen haben gewonnen,

014 pek $[<<f>N E I: N ;>]$

015 sus $[<<f>N E I: N ;>]$ 


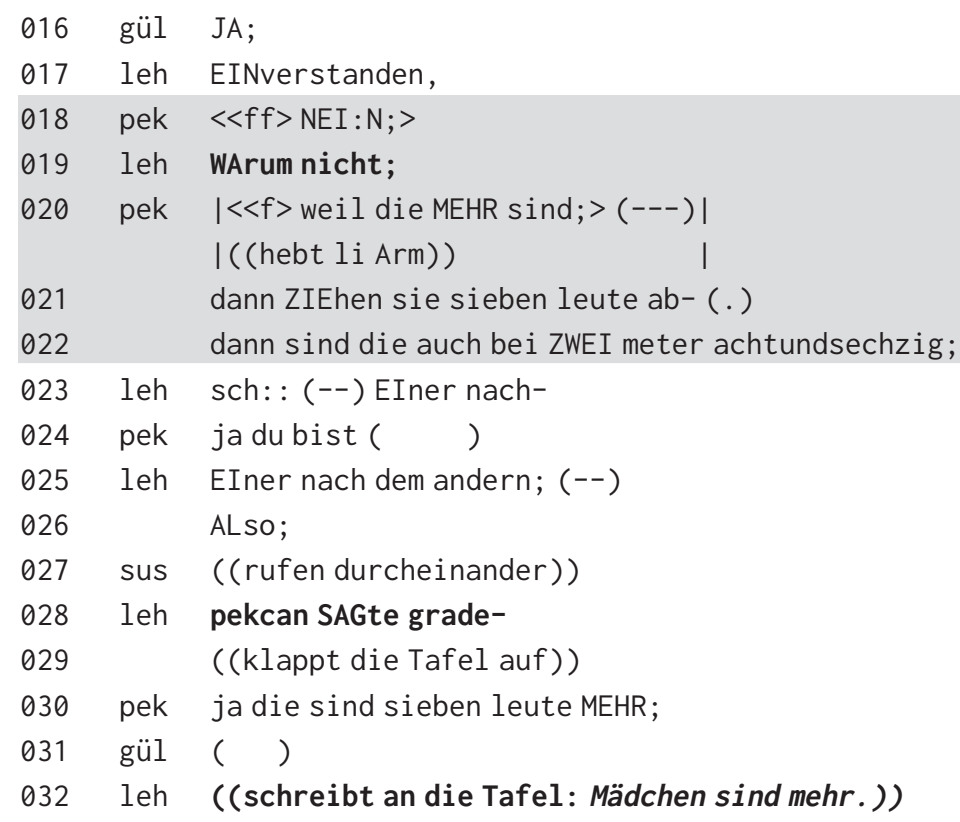

Von den spontan geäußerten Positionen greift der Lehrer diejenige auf, die die Mädchen als Gewinner postuliert (Z. 13) und schließt - den Dissens scheinbar nicht wahrnehmend - eine Entscheidungsfrage an (Z. 17: „EINverstanden,“). Daraufhin äußert Pekcan als erster einen Widerspruch (Z. 18: „«<f > NEI:N; >“). Damit ist der Dissens, der zwar schon an den überlappend hereingerufenen Äußerungen ablesbar war, nun in geordneter und für alle wahrnehmbarer Weise etabliert. In unmittelbarem Anschluss richtet der Lehrer nun eine warum-Frage (Z. 19) an Pekcan. Diese gattungsindizierende Frage teilt Pekcan nicht nur offiziell das Rederecht $\mathrm{zu}$, sondern setzt zugleich auch einen impliziten globalen Zugzwang zum Argumentieren und etabliert damit eine Begründungspflicht (Heller 2012), deren Erfüllung in der Regel die Produktion eines Äußerungspakets mit sich bringt. Pekcan bedient den globalen Zugzwang, indem er ein solches Äußerungspaket (Z. 20-22) produziert: Er nennt einen Grund (, $\ll<f f>$ weil die MEHR sind; >") und elaboriert diesen, indem er ausführt, welche Konsequenzen die Herstellung gleicher Bedingungen hätte: Wenn sieben Mädchen abgezogen werden (und somit eine gleiche Anzahl an Jungen und Mädchen hergestellt wird), dann gäbe es auch keinen Gewinner. Freilich ist in Pekcans Konditionalkonstruktion die Protasis nicht durch wenn markiert, sondern durch dann. Zudem wird das Ziel der Herstellung gleicher Gruppengrößen nicht eigens expliziert, sondern muss von den Rezipienten selbstständig erschlossen werden. Dennoch kann aber der argumentative Zugzwang als angemessen kontextualisiert 
gelten. Dementsprechend behandelt der Lehrer Pekcans Beitrag als funktional und stellt ihn zur Disposition, indem er dessen Kern für alle sichtbar an der Tafel fixiert (Z. 28-32). Pekcan ist also in dieser Sequenz erstmals vor die globale Anforderung des Begründens gestellt, und zwar in unmittelbarem Anschluss an seine Positionsbekundung. Es reichte ein implizit formulierter globaler Zugzwang mit gattungsindizierender Frage, um ihn zum Begründen zu veranlassen.

\section{Pekcan II: Vergrößern und Verkleinern der diskursiven Anforderung}

Pekcan schaltet sich nun noch an einer weiteren Stelle in die Argumentation ein, die insbesondere mit Blick auf die Feinabstimmung der Unterstützung für die Kontextualisierung argumentativer Zugzwänge interessant ist. In dieser zweiten Sequenz hat sich der Dissens verschoben, und zwar durch einen Vorschlag, der ebenfalls von Pekcan stammte: Er hatte angeregt, gleich große Gruppen durch einfaches Abzählen herzustellen (Z. 113-114). Nachdem der Vorschlag bereits von Bernadette Zustimmung erfahren hatte (Z. 123-124), wird nun ein weiterer Dissens etabliert bezüglich der Frage, welche sieben Mädchen nicht in den Vergleich einbezogen werden sollen (Z. 125-156).

Beispiel (5) 01-5-MU-07-22492909_Wer hat denn jetzt gewonnen_2

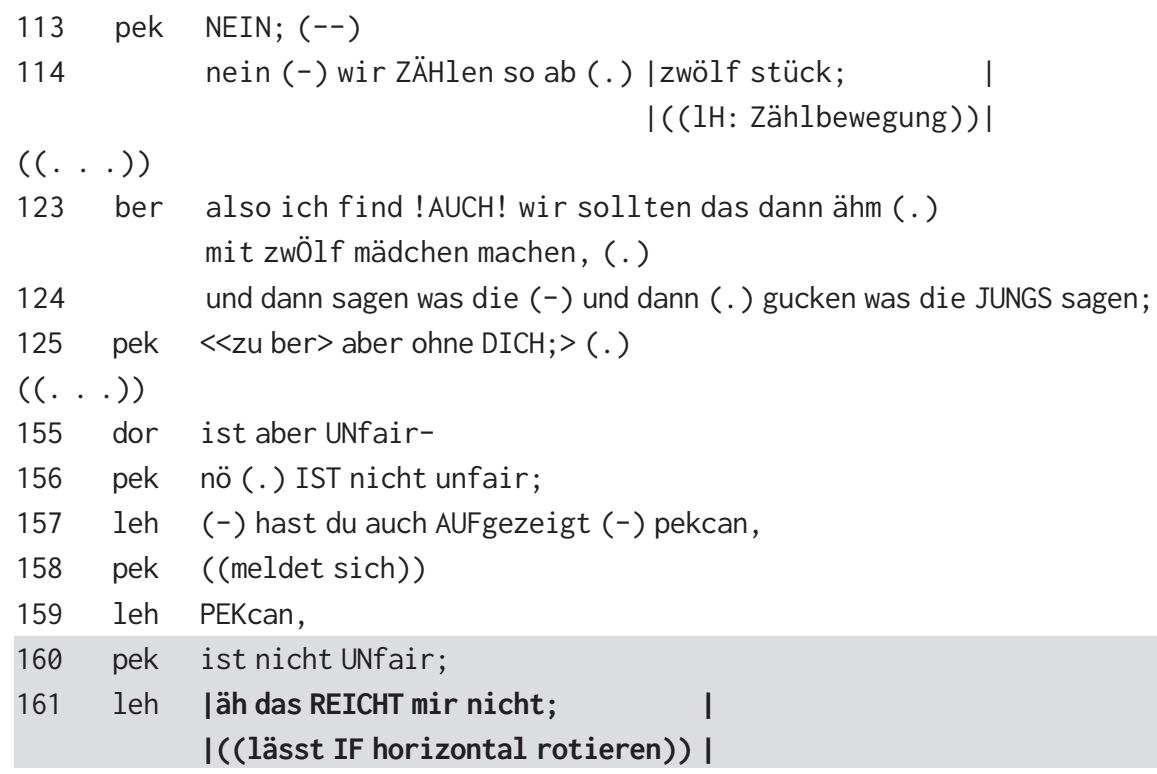




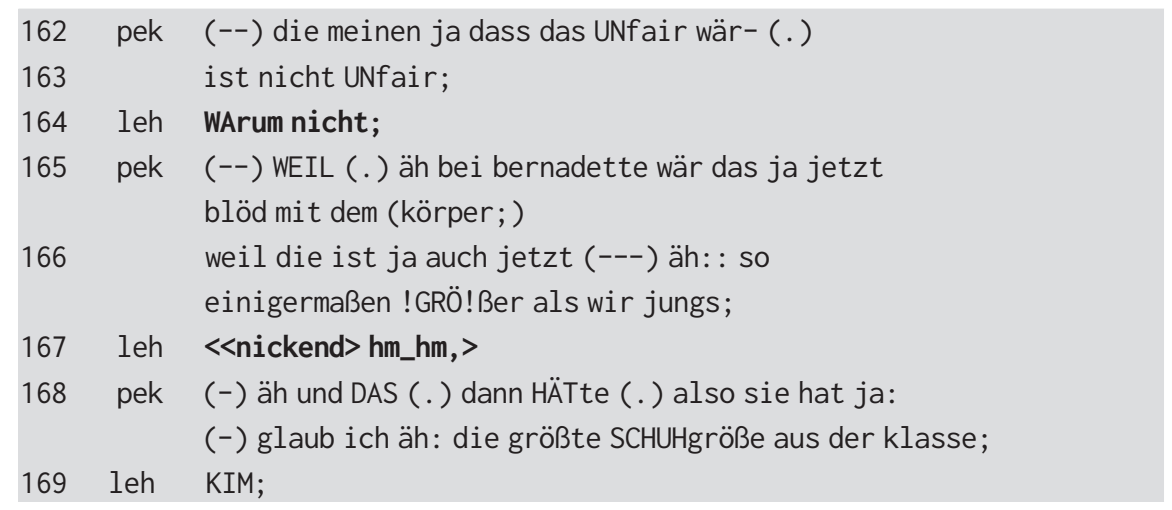

Die Vorrednerin Mona hatte bereits selbst eine Begründung ihrer Position produziert (nicht im Transkript gezeigt); die Begründungspflicht wird danach nicht noch einmal durch entsprechende lehrerseitige Fragen etabliert, sondern muss aus dem sequenziellen Kontext geschlossen werden. Pekcan äußert nun als Widerspruch zu Doros Einwand (Z. 155), dass die Nichtberücksichtigung von Bernadette unfair sei, ohne diesen Widerspruch zu begründen (Z. 156). Er schließt aus dem sequenziellen Kontext also nicht, dass eine Begründungspflicht besteht. Der Mathematiklehrer reagiert auf dieses Kontextualisierungsproblem, indem er zunächst nur metadiskursiv anzeigt, dass der Beitrag nicht seinen kommunikativen Erwartungen entspricht: „äh das REICHT mir nicht;“ (Z. 161). Dabei wendet er sich Pekcan zu und zeigt mit einer Geste - dem Rotieren seines horizontal ausgestreckten Zeigefingers - an, dass die Äußerung fortgesetzt werden soll. Das Rederecht geht nun nicht an einen anderen Schüler, sondern an Pekcan zurück. Dies ist die Voraussetzung dafür, dass Pekcan im Folgenden eine feinabgestimmte Lerngelegenheit für die Kontextualisierung argumentativer Zugzwänge erhalten kann. Zunächst ist zu beobachten, dass der Lehrer mit der metadiskursiven Aussage die Anforderung im Vergleich zur ersten Sequenz angehoben hat: Pekcan ist nun gefordert selbst zu schließen, was genau er tun soll. Er kontextualisiert den Kommentar des Lehrers jedoch zunächst als Aufforderung zu einer referentiell eindeutigeren und syntaktisch vollständigeren Äußerung (Z. 162-163). Im Anschluss an diese noch nicht adäquate Kontextualisierung lässt der Lehrer - erneut an Pekcan adressiert - eine warum-Frage folgen, mittels der er seine kommunikative Erwartung spezifiziert. Mit der warum-Frage hat der Lehrer das Ausmaß der Unterstützung nun wieder genau auf das Kompetenzniveau abgestimmt, das Pekcan schon in der ersten Sequenz gezeigt hatte. Auf eine umfänglichere Unterstützung in Form einer Explikation des Zugzwangs wird hingegen verzichtet. Für Pekcan ist diese Unterstützung nun ausreichend, um zu erkennen, dass eine Begründung gefordert ist. In seiner Begründung veranschaulicht er am Beispiel Bernadettes (der größten Schülerin der Klasse), dass nicht willkürlich 
bestimmte Kinder für den Vergleich ausgewählt werden dürfen (jedoch noch ohne dies in generalisierender Weise zu thematisieren). Der Lehrer zeigt währenddessen mittels eines continuers an, dass Pekcan weiterhin das Rederecht zusteht; zugleich gibt er damit eine Rückmeldung, dass die kommunikative Erwartung nun angemessen kontextualisiert wurde.

In der zweiten Begründungssequenz hat der Lehrer also zunächst die Anforderung zu steigern versucht, indem er Pekcan zumutet, die kommunikative Erwartung selbstständig aus dem sequenziellen Kontext zu schließen. Nachdem dies nicht gelingt, gewährt er eine feinabgestimmte Unterstützung, indem er die Formulierung des Zugzwangs an das zuvor gezeigte Kompetenzniveau anpasst, aber keine darüberhinausgehende Hilfestellung in Form einer Explikation des Zugzwangs gibt. Insgesamt zielt das Verfahren des Lehrers darauf, Pekcan an den autonomen Umgang mit konditionellen Relevanzen heranzuführen.

\section{Pekcan III: Erneutes Anheben der Anforderung in der Folgestunde}

In der folgenden Mathematikstunde wird der Argumentationsgegenstand des vorigen Tages noch einmal aufgegriffen. Ausgangspunkt ist nun die Positionsbekundung der fiktiven Figur Merve aus dem Mathematikbuch (s. Abb. 2), in der diese in unzulässiger Weise von einem Einzelfall generalisiert. Zu dieser Position sollen die Schülerinnen und Schüler Stellung beziehen. Im Unterschied zu der vorangegangenen Stunde sind diese nun nicht mehr selbst in den Entscheidungsprozess involviert, sondern argumentieren hypothetisch und aus größerer Distanz.

\section{Wie kann man Zahlenangaben von zwei Gruppen vergleichen?}

2 Vergleichen von zwei Gruppen

In der Klasse von Merve wurde untersucht, ob die Jungen oder die Mädchen die größeren Füße haben.

Warum sollte man nicht so wie Merve vorgehen, um zwei Gruppen zu vergleichen?

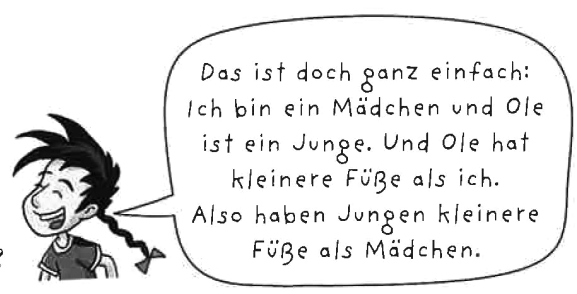

Abb. 2: Aufgabe aus dem Mathematikbuch (Barzel et al. 2012: 21).

Vorangegangen ist dem in Beispiel (6) gezeigten Ausschnitt, dass eine Schülerin die entsprechende Sprechblase und die anschließende Frage vorliest. Der 
Mathematiklehrer reformuliert nun die Äußerung der fiktiven Figur Merve mittels einer direkten Redewiedergabe (Z. 2-7) und schaut dann erwartungsvoll in die Klasse (Z. 8). Es wird weder ein expliziter noch ein impliziter Zugzwang gesetzt; vielmehr sind die Schülerinnen und Schüler selbst gefordert zu erkennen, dass sie argumentativ Stellung nehmen sollen.

Beispiel (6) 01-5-MU-08-00280229_Merves Aussage

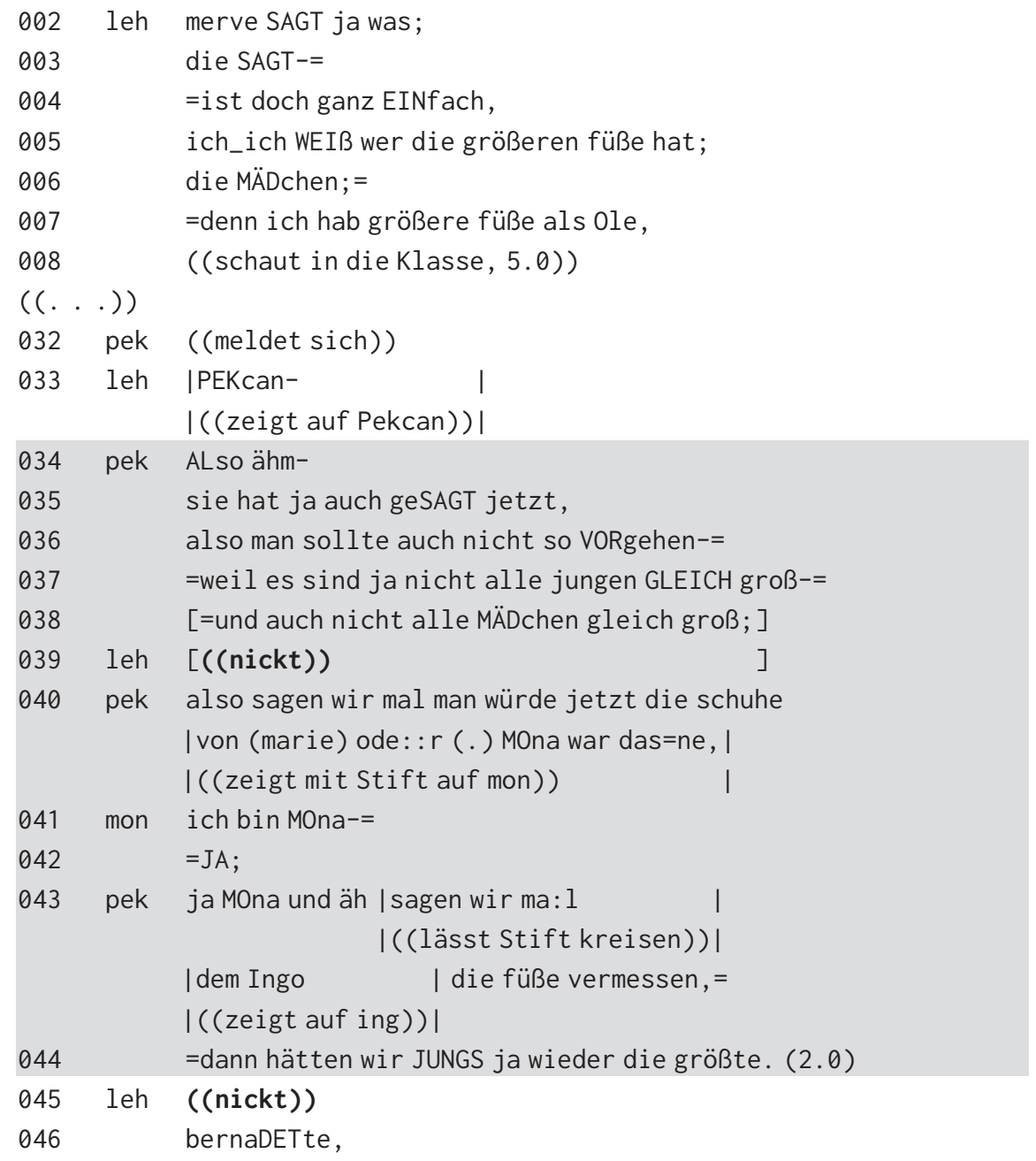

Als der Lehrer Pekcan das Rederecht übergibt, sieht er davon ab, den Zugzwang $\mathrm{zu}$ explizieren oder $\mathrm{zu}$ spezifizieren; er hat somit die Anforderung an die 
Kontextualisierung erneut angehoben. Anders als in der zweiten Sequenz gelingt es Pekcan nun, aus dem Kontext auf die bestehende Begründungspflicht zu schließen. Dass seine Begründung nun deutlich autonomer kontextualisiert ist, zeigt sich u. a. daran, dass er gleich zu Beginn durch den Diskursmarker also (Z. 34) ein längeres Äußerungspaket projiziert. Des Weiteren nimmt er Bezug auf seine Vorrednerin (Z. 35-37) und stellt auf diese Weise eine turnübergreifende Kohärenz zwischen den Argumenten her. Damit kontextualisiert er autonom, dass sein Beitrag als Ergänzung zu (und nicht etwa als Erwiderung auf) Carlas Begründung zu verstehen ist. Durch Rezeptionssignale (Continuer und Nicken) bestätigt der Lehrer die Angemessenheit der Kontextualisierung und gewährt Raum für die weitere Vertextung (Z. 39). Daraufhin baut Pekcan seine Vertextung aus, indem er ein fiktives Szenario (Brünner \& Gülich 2002; Heller in diesem Band) etabliert, das er mit der sedimentierten Projektor-Konstruktion (Couper-Kuhlen \& Selting 2018: 487) „also sagen wir mal“ ebenfalls explizit einführt. Neben der autonomeren Kontextualisierung zeigen sich somit auch Unterschiede in der Vertextung und Markierung: Das fiktive Szenario wird in einer Weise vertextet, die die Konsequenzen einer willkürlichen Auswahl von Vergleichselementen nachvollziehbar veranschaulicht. Zudem wird der exemplarische Status durch den Konjunktiv und die nun klarer markierte Konditionalkonstruktion verdeutlicht. Wenngleich sich auch in den Dimensionen der Vertextung und Markierung Übungseffekte zeigen, so ist doch die Unterstützung des Mathematiklehrers hier zunächst vornehmlich auf die Kontextualisierung bezogen.

Der Längsschnitt zeigt also, dass die feinabgestimmte Unterstützung in der ersten Stunde und die ebenfalls feinabgestimmte Anhebung der diskursiven Anforderungen in der Folgestunde dazu führen, dass Pekcan konditionelle Relevanzen für das Argumentieren autonomer kontextualisiert.

\section{Finetuning als konstitutives Element diskurserwerbsförderlichen Lehrerhandelns}

Der vorliegende Beitrag hat das Konzept des individualisierten Zuschnitts diskursiver Anforderung und Unterstützung anhand querschnittlicher und längsschnittlicher Zugriffsweisen auszuschärfen versucht. Unter Finetuning diskurserwerbsförderlichen Lehrerhandelns in Unterrichtsinteraktionen verstehen wir das kontext- und lernersensitive Setzen lokaler und globaler Zugzwänge zur Involvierung einzelner Schülerinnen und Schüler in die Bearbeitung komplexer fachlicher und diskursiver Aufgaben. Dabei werden gattungsbezogene Anforderungen (zum Beschreiben, Erklären, Argumentieren usw.) innerhalb einer 
Sequenz oder über Sequenzen hinweg so verkleinert bzw. vergrößert, dass sie für Schülerinnen und Schüler mit ganz unterschiedlichen Ausgangsbedingungen bearbeitbar gemacht werden. Solche Prozesse des Forderns und Unterstützens manifestieren sich Zug-um-Zug in der kokonstruktiven Hervorbringung einer spezifischen Interaktionssequenz zwischen einer Lehrperson und einem Lernenden oder über mehrere Sequenzen, verteilt über Tage oder Wochen, hinweg. Finetuning diskurserwerbsförderlichen Lehrerhandelns erfordert somit nicht nur die sinnvolle Nutzung von Gattungen für Zwecke der Wissenskonstruktion und -vermittlung, sondern auch ein aufmerksames und verständigungsorientiertes Zuhören und Reagieren auf schülerseitig eingebrachte Äußerungen. Tabelle 2 führt zentrale Finetuning-Verfahren auf.

Tab. 2: Finetuning diskurserwerbsförderlichen Lehrerhandelns.

\section{Finetuning diskurserwerbsförderlichen Lehrerhandelns}

(1) Kalibrieren des Anforderungsniveaus beim initialen Setzen von Zugzwängen

a. Gattungen mit sequenzierender Vertextung (Berichten, Beschreiben, Erklären-Wie) vs. Gattungen mit integrierender Vertextung (Erklären-Was, -Warum, Argumentieren)

b. Implizite (aus dem sequenziellen Kontext zu schließende oder mit einem gattungsindizierenden Frageadverb versehene) oder explizite globale Zugzwänge

c. Persönlich oder an die Gruppe adressierte globale Zugzwänge

(2) Readressieren und Modifizieren von globalen Zugzwängen

a. Verkleinern einer diskursiven Anforderung

- Lokalisieren

- Explizieren

- Gattungswechsel (bspw. einen Zugzwang zum Erklären-Warum in einen Zugzwang zum Erklären-Wie oder Berichten modifizieren

b. Vergrößern einer diskursiven Anforderung

- Wiedereinsetzen des globalen Zugzwangs

- im Anschluss an einen lokalen Zugzwang einen globalen Zugzwang setzen

- Gattungswechsel (im Anschluss an einen Zugzwang zum Berichten/ Beschreiben einen Zugzwang zum Erklären oder Argumentieren setzen)

(3) Adressierung von mehr oder weniger Zuhöreraktivitäten in Form von

a. fokussierenden/refokussierenden Zuhöreraktivitäten

b. mitkonstruierenden Zuhöreraktivitäten (z. B. Reformulierungen, Elaborierungsfragen und klärende Nachfragen)

c. verständnisanzeigenden Zuhöreraktivitäten (z. B. Continuer)

d. interaktiv eingebetteten sprachlich-diskursiven Modellen

Die hier vorgestellten Analysen zeigen, dass ein solcher individualisierter Zuschnitt diskursiver Anforderung und Unterstützung in Unterrichtsgesprächen grundsätzlich möglich ist. Befunde der linguistischen Unterrichtsforschung 
und Lehr-Lernforschung weisen allerdings auch darauf hin, dass dieser nicht von allen Lehrkräften und auch nicht in Bezug auf alle Schülerinnen und Schüler realisiert wird (Heller 2017; Quasthoff \& Prediger 2017). Untersuchungen zur Wirksamkeit von Professionalisierungsmaßnahmen in diesem Bereich (Pfister et al. 2015) legen den Schluss nahe, dass Verfahren, die eine kontext- und lernersensitive Weiterarbeit mit Schülerbeiträgen beinhalten und somit über schematisch einzusetzende Techniken oder "moves“ (Michaels \& O’Connor 2013) hinausgehen, auf Seiten der Lehrpersonen langwierige Aneignungsprozesse erfordern. Ein dringendes Desiderat besteht somit darin, geeignete Methoden für die Professionalisierung im Bereich individualisierter Diskurserwerbsförderung $\mathrm{zu}$ entwickeln und $\mathrm{zu}$ untersuchen, welche Faktoren deren lehrerseitige Aneignung und Nutzung bedingen.

Daran schließt sich ein weiteres Desiderat an: Um differenzierte und belastbare Aussagen zur Wirksamkeit eines individualisierten Zuschnitts diskursiver Anforderungen und Unterstützungsaktivitäten für unterschiedliche Schülergruppen treffen zu können, sind im Anschluss an den hier genutzten rekonstruktiven $\mathrm{Zu}$ griff quantifizierende Zugänge notwendig. Derzeit existieren jedoch noch keine interaktionssensiblen Kodiersysteme, die a) empirisch basierte, gesprächsanalytisch rekonstruierte Verfahren berücksichtigen und es b) erlauben, die Feinabstimmung zwischen Schüler- und Lehreräußerungen zu erfassen. Es sind mit anderen Worten Kodiersysteme zu entwickeln, die die Interaktivität und Prozessualität von Feinabstimmung zumindest in Ansätzen berücksichtigen und es ermöglichen, Folgen von Zugzwängen, Schülerbeiträgen und lehrerseitiger Unterstützung kodierend $\mathrm{zu}$ erfassen und die Zusammenhänge unterschiedlicher Muster solcher Folgen mit der Kompetenzentwicklung von Schülerinnen und Schülern über die Zeit zu vergleichen. Die Analysen in diesem Beitrag jedenfalls geben Anlass zur Annahme, dass das Finetuning lehrerseitiger Unterstützungsaktivitäten im Unterricht eine ganz zentrale Rolle für die Schaffung sprachlich-diskursiver Lerngelegenheiten - gerade in inklusiven Klassen - spielt.

\section{Literatur}

Barzel, Bärbel, Stephan Hußmann, Timo Leuders \& Susanne Prediger (Hrsg.), Mathewerkstatt. Klasse 5. Cornelsen: Berlin.

Böttcher, Wolfgang (1994): Grammatiksozialisation in Schule, Hochschule und Referendarausbildung. Beiträge zur Lehrerbildung 12 (2), 170-186.

Bruner, Jerome (1983): Child's talk. Learning to use language. New York: Norton. 
Bruner, Jerome \& Virginia Sherwood (1976): Peek-a-boo and the learning of rule structures. In Jerome Bruner, Alison Jolly \& Kathy Sylva (Hrsg.), Play - Its role in development and evolution, 277-287. New York: Basic-Books.

Brünner, Gisela \& Elisabeth Gülich (2002): Verfahren der Veranschaulichung in der ExpertenLaien-Kommunikation. In Gisela Brünner \& Elisabeth Gülich (Hrsg.), Krankheit verstehen. Interdisziplinäre Beiträge zur Sprache in Krankheitsdarstellungen, 17-93. Bielefeld: Aisthesis Verlag.

Clark, Eve (2018): Conversation and Language Acquisition. A Pragmatic Approach. Language Learning and Development 14 (3), 170-185.

Couper-Kuhlen, Elizabeth \& Margret Selting (2018): Interactional linguistics. Studying language in social interaction. Cambridge: Cambridge University Press.

Cross, Toni \& Jenny Morris (1980): Linguistic Feedback and Maternal Speech: Comparisons of Mothers Addressing Infants, One-Year-Olds and Two-Year-Olds. First Language 1 (2), 98-121.

Cross, Toni, Terry Nienhuys \& Maggie Kirkman (1985): Parent-child interaction with receptively disabled chhildren: some determinants of maternal speech style. In Keith Nelson (Hrsg.), Children's Language, 247-290. New York: Psychology Press.

Doughty, Catherine (1993): Fine tuning of feedback by competent speakers to language learners. In James Alatis (Hrsg.), Strategic interaction and language acquisition. Theory, practice, and research, 96-108. Washington, DC: Georgetown Univ. Press.

Ehlich, Konrad \& Jochen Rehbein (1986): Muster und Institution. Untersuchungen zur schulischen Kommunikation. Tübingen: Narr.

Ferguson, Charles (1977): Baby Talk as Simplified Register. In Catherine Snow \& Charles Ferguson (Hrsg.), Talking to Children. Language Input and Acquisition, 219-235. Cambrigde u. a.: Cambridge University Press.

Fischer, Kerstin (2016): Designing speech for a recipient. The roles of partner modeling, alignment and feedback in so-called 'simplified registers'. Amsterdam: John Benjamins.

Gallaway, Clare \& Brian Richards (Hrsg.) (1994): Input and interaction in language acquisition. Cambridge: Cambridge University Press.

Garnica, Olga (1977): Some Prosodic and Paralinguistic Features of Speech to Young Children. In Catherine Snow \& Charles Ferguson (Hrsg.), Talking to Children: Language Input and Acquisition, 63-88. Cambridge: Cambridge University Press.

Gibbons, Pauline (2002): Scaffolding Language, Scaffolding Learning. Teaching Second Language Learners in the Mainstream Classroom. Portsmouth, NH: Heinemann.

Hammond, Jenny \& Pauline Gibbons (2005): Putting scaffolding to work: The contribution of scaffolding in articulating ESL education. Prospect 20 (1), 6-30.

Harren, Inga (2015): Fachliche Inhalte sprachlich ausdrücken lernen. Sprachliche Hürden und interaktive Vermittlungsverfahren im naturwissenschaftlichen Unterrichtsgespräch in der Mittel- und Oberstufe. Radolfzell: Verlag für Gesprächsforschung.

Hausendorf, Heiko \& Uta Quasthoff (1996/2005): Sprachentwicklung und Interaktion. Eine linguistische Studie zum Erwerb von Diskursfähigkeiten. Radolfzell: Verlag für Gesprächsforschung. Neu aufgelegt: http://www.verlag-gespraechsforschung.de/2005/ quasthoff.htm. (Letzter Aufruf am 27.06.2020) Radolfzell: Verlag für Gesprächsforschung.

Heller, Vivien (2012): Kommunikative Erfahrungen von Kindern in Familie und Unterricht. Passungen und Divergenzen. Tübingen: Stauffenburg.

Heller, Vivien (2017): Lerngelegenheiten für bildungssprachliche Kompetenzen: Wie partizipieren DaZ-Lerner am Erklären und Argumentieren im Unterricht? In Isabel Fuchs, 
Stefan Jeuk \& Werner Knapp (Hrsg.), Mehrsprachigkeit: Spracherwerb, Unterrichtsprozesse, Schulentwicklung, 165-182. Stuttgart: Fillibach bei Klett.

Heller, Vivien \& Antje Krah (2015): Wie Eltern und Kinder argumentieren. Interaktionsmuster und ihr erwerbssupportives Potenzial im längsschnittlichen Vergleich. Mitteilungen des Deutschen Germanistenverbandes 62 (1), 5-20.

Heller, Vivien \& Miriam Morek (2015): Unterrichtsgespräche als Erwerbskontext: Kommunikative Gelegenheiten für bildungssprachliche Praktiken erkennen und nutzen. leseforum.ch (3), 1-23.

Heller, Vivien, Uta Quasthoff, Susanne Prediger \& Anna Vogler (2017): Bildungssprachliche Praktiken aus professioneller Sicht: Wie deuten Lehrende Schülererklärungen und -begründungen? In Bernt Ahrenholz, Britta Hövelbrinks \& Claudia Schmellentin (Hrsg.), Fachunterricht und Sprache in schulischen Lehr-/Lernprozessen, 139-160. Tübingen: Narr.

Heller, Vivien \& Katharina Rohlfing (2017): Reference as an Interactive Achievement. Sequential and Longitudinal Analyses of Labeling Interactions in Shared Book Reading and Free Play. Frontiers in Psychology 8 (139), 1-19.

Hitzler, Sarah (2013): Recipient Design in institutioneller Mehrparteieninteraktion. Gesprächsforschung - Online-Zeitschrift zur verbalen Interaktion 14, 110-132.

Hövelbrinks, Britta (2014): Bildungssprachliche Kompetenz von einsprachig und mehrsprachig aufwachsenden Kindern. Eine vergleichende Studie in naturwissenschaftlicher Lernumgebung des ersten Schuljahres. Weinheim, Basel: Beltz Juventa.

Jefferson, Gail (1978): Sequential aspect of story-telling. In Jim Schenkein (Hrsg.), Studies in the organization of conversational interaction, 219-248. New York: Academic Press.

Kern, Friederike \& Uta Quasthoff (2007): Familiale Interaktionsmuster und kindliche Diskursfähigkeit: Mögliche Auswirkungen interaktiver Stile auf diskursive Praktiken und Kompetenzen bei Schulkindern. In Heiko Hausendorf (Hrsg.), Gespräch als Prozess. Linguistische Aspekte der Zeitlichkeit verbaler Interaktion, 277-306. Tübingen: Narr.

Kleinschmidt-Schinke, Katrin (2018): Die an Schüler/-innen gerichtete Sprache (SgS). Studien zur Veränderung der Lehrer/-innensprache von der Grundschule bis zur Oberstufe. Berlin: De Gruyter.

Koole, Tom \& Ed Elbers (2014): Responsiveness in teacher explanations: A conversation analytical perspective on scaffolding. Linguistics and Education 26, 57-69.

Lerner, Gene (1995): Turn design and the organization of participation in instructional activities. Discourse Processes 19 (1), 111-131.

McCabe, Allyssa \& Carole Peterson (1991): Getting the story. A Longitudinal Study of Parental Styles in Eliciting Narratives and Developing Narrative Skill. In Allyssa McCabe \& Carole Peterson (Hrsg.), Developing narrative structure, 217-254. Hillsdale, N.J: L. Erlbaum.

Michaels, Sarah \& Mary O'Connor, Megan Williams Hall \& Lauren Resnick (2013): Accountable Talk Sourcebook: For Classroom Conversation that Works.

Morek, Miriam (2012): Kinder erklären. Interaktionen in Familie und Unterricht im Vergleich. Tübingen: Stauffenburg.

Morek, Miriam (2016): Formen mündlicher Darstellung in situ: Zur Komplexität von Diskursanforderungen in Unterrichtsgesprächen. In Ulrike Behrens \& Olaf Gätje (Hrsg.), Mündliches und schriftliches Handeln im Deutschunterricht. Wie Themen entfaltet werden, 95-131. Frankfurt a.M.: Peter Lang. 
Morek, Miriam, Vivien Heller \& Uta Quasthoff (2017): Erklären und Argumentieren. Modellierungen und empirische Befunde im Rahmen der linguistischen Erwerbs- und Unterrichtsforschung. In Iris Meißner \& Eva Wyss (Hrsg.), Begründen - Erklären Argumentieren. Konzepte und Modellierungen in der Angewandten Linguistik, 11-46. Tübingen: Stauffenburg.

Ninio, Anat \& Jerome Bruner (1978): The achievement and antecedents of labelling. Journal of child language 5 (1), 1-15.

Ochs, Elinor (2002): Becoming a speaker of culture. In Claire Kramsch (Hrsg.), Language acquisition and language socialization. Ecological perspectives, 99-120. London, New York: Continuum.

Pehmer, Ann-Kathrin, Alexander Gröschner \& Tina Seidel (2015): Fostering and scaffolding student engagement in productive classroom discourse. Teachers' practice changes and reflections in light of teacher professional development. Learning, Culture and Social Interaction 7, 12-27.

Pfister, Mirjam, Elisabeth Moser Opitz \& Christine Pauli (2015): Scaffolding for mathematics teaching in inclusive primary classrooms. A video study. ZDM Mathematics Education 47 (7), 1079-1092.

Quasthoff, Uta (2011): Diskurs- und Textfähigkeiten: Kulturelle Ressourcen ihres Erwerbs. In Ludger Hoffmann, Kerstin Leimbrink \& Uta Quasthoff (Hrsg.), Die Matrix der menschlichen Entwicklung, 210-251. Berlin: De Gruyter.

Quasthoff, Uta (2012): Globale und lokale Praktiken in unterschiedlichen diskursiven Genres: Wie lösen L2-Sprecher globale Anforderungen bei eingeschränkter sprachstruktureller Kompetenz im Deutschen? In Heike Roll \& Andrea Schilling (Hrsg.), Mehrsprachiges Handeln im Fokus von Linguistik und Didaktik, 47-65. Duisburg: Universitätsverlag Rhein-Ruhr.

Quasthoff, Uta (2015): Ko-Konstruktion in Erwachsenen-Kind-Interaktionen: Membership und der Erwerb von sprachlicher Kompetenz. In Ulrich Dausendschön-Gay, Elisabeth Gülich \& Ulrich Krafft (Hrsg.), Ko-Konstruktionen in der Interaktion. Die gemeinsame Arbeit an Äußerungen und anderen sozialen Ereignissen, 287-312. Bielefeld: transcript.

Quasthoff, Uta \& Susanne Prediger (2017): Fachbezogene Unterrichtsdiskurse zu Beginn der weiterführenden Schule - Interdisziplinäre Untersuchungen zur Unterstützung von sprachlichem und fachlichem Lernen. In Arne Krause, Gesa Lehmann, Winfried Thielmann \& Caroline Trautmann (Hrsg.), Form und Funktion, 625-644. Tübingen: Stauffenburg Verlag.

Ricart Brede, Julia (2011): Videobasierte Qualitätsanalyse vorschulischer Sprachfördersituationen. Freiburg im Breisgau: Fillibach.

Sacks, Harvey, Emanuel Schegloff \& Gail Jefferson (1974): A Simplest Systematics for the Organization of Turn-Taking for Conversation. Language 50 (4), 696-735.

Schegloff, Emanuel \& Harvey Sacks (1973): Opening Up Closings. Semiotica 8, 289-327.

Schieffelin, Bambi (1990): The give and take of everyday life: Language socialization of Kaluli children. Cambridge, UK: Cambridge University Press.

Selting, Margret, Peter Auer, Dagmar Barth-Weingarten, Jörg Bergmann, Pia Bergmann, Karin Birkner et al. (2009): Gesprächsanalytisches Transkriptionssystem 2 (GAT 2). Gesprächsforschung - Online-Zeitschrift zur verbalen Interaktion (10), 292-341.

Smit, Jantien, Henriëtte van Eerde \& Arthur Bakker (2013): A conceptualisation of whole-class scaffolding. British Educational Research Journal 39 (5), 817-834.

Smit, Jantien \& Dolly van Eerde (2013): What counts as evidence for the long-term realisation of whole-class scaffolding? Learning, Culture and Social Interaction 2 (1), 22-31. 
Snow, Catherine (1972): Mothers' Speech to Children Learning Language. Child Development 43 (2), 549-565.

Snow, Catherine (1977): Mother's Speech Research: from Input to Interaction. In Catherine Snow \& Charles Ferguson (Hrsg.), Talking to Children. Language Input and Acquisition, 31-49. Cambrigde: Cambridge University Press.

Snow, Catherine, Rivka Perlmann \& Debra Nathan (1987): Why Routines are Different. Toward a Multiple-Factors Model of the Relation between Input and Language Acquisition. In Catherine Nelson, E. Keith \& Anne van Kleeck (Hrsg.), Children's Language, 65-97. Hillsday, NJ: Erlbaum.

Sokolov, Jeffrey (1993): A local contingency analysis of the fine-tuning hypothesis. Developmental Psychology, 29, 1008-1023.

van de Pol, Janneke, Monique Volman, Frans Oort \& Jos Beishuizen (2015): The effects of scaffolding in the classroom: support contingency and student independent working time in relation to student achievement, task effort and appreciation of support. Instructional Science 43 (5), 615-641.

van Driel, Sharisse, Esther Slot \& Arthur Bakker (2018): A Primary Teacher Learning to Use Scaffolding Strategies to Support Pupils' Scientific Language Development. European Journal of STEM Education 3 (2), 05.

Vygotsky, Lev (1978): Mind in society. The development of higher psychological processes. Cambridge, Mass.: Harvard University Press.

Wood, David, Jerome Bruner \& Gail Ross (1976): The role of tutoring in problem solving. Journal of Child Psychology 17, 89-100.

Zevenbergen, Andrea, Ashley Holmes, Ewa Haman, Nichole Whiteford, \& Shelly Thielges (2016): Variability in mothers' support for preschoolers' contributions to co-constructed narratives as a function of child age. First Language 36 (6), 601-616. 